\title{
Radial expansion for spinning conformal blocks
}

\author{
Miguel S. Costa, ${ }^{a, b}$ Tobias Hansen, ${ }^{a}$ João Penedones ${ }^{a, b, c}$ and Emilio Trevisani ${ }^{a}$ \\ ${ }^{a}$ Centro de Fisica do Porto, Universidade do Porto, \\ Porto, Portugal \\ ${ }^{b}$ Theory Division, Department of Physics, \\ CERN,CH-1211 Genève 23, Switzerland \\ ${ }^{c}$ Fields and Strings Laboratory, Institute of Physics, \\ EPFL, CH-1015 Lausanne, Switzerland \\ E-mail: miguelc@fc.up.pt, thansen@fc.up.pt, jpenedones@gmail.com, \\ emiliotrevisani@yahoo.it
}

ABSTRACT: This paper develops a method to compute any bosonic conformal block as a series expansion in the optimal radial coordinate introduced by Hogervorst and Rychkov. The method reduces to the known result when the external operators are all the same scalar operator, but it allows to compute conformal blocks for external operators with spin. Moreover, we explain how to write closed form recursion relations for the coefficients of the expansions. We study three examples of four point functions in detail: one vector and three scalars; two vectors and two scalars; two spin 2 tensors and two scalars. Finally, for the case of two external vectors, we also provide a more efficient way to generate the series expansion using the analytic structure of the blocks as a function of the scaling dimension of the exchanged operator.

KeYwords: Conformal and W Symmetry, Field Theories in Higher Dimensions

ArXiv EPrint: 1603.05552v2 


\section{Contents}

1 Introduction 1

2 Review of the scalar conformal block $\quad 2$

2.1 A natural series expansion 4

2.2 A recurrence relation for the expansion coefficients 5

3 Spinning conformal blocks $\quad 9$

3.1 General formalism and example of one vector and three scalars 9

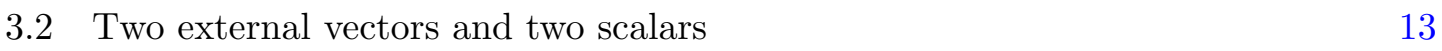

$\begin{array}{lll}3.3 & \text { Two external spin } 2 \text { operators and two scalars } & 19\end{array}$

4 Recursion relation from analytic structure in $\Delta \quad 21$

4.1 Recursion relation for two external vectors and two scalars 22

5 Conclusion $\quad 24$

$\begin{array}{ll}\text { A Differential equations in Gegenbauer basis } & 27\end{array}$

B Details on the recursion relation from the analytic structure in $\Delta \quad 29$

B.1 The residues $R_{A} \quad 30$

B.2 Conformal block at large $\Delta \quad 34$

B.3 The OPE limit of conformal blocks 35

$\begin{array}{ll}\text { C Differential operators method } & 37\end{array}$

\section{Introduction}

The conformal bootstrap is opening a new window into quantum field theory beyond perturbation theory [1-34]. In this paper we present a method to construct any bosonic conformal block (CB), an essential ingredient to formulate the conformal bootstrap equations.

Constructing general CBs is still an open problem. The exceptions are for three [35] and four [36] dimensions. ${ }^{1}$ In these cases it was possible to construct the full set of seed blocks which are sufficient to determine all CBs by acting with differential operators [26, 38, 39]. However for general spacetime dimensions the problem is far from being solved. In fact, when the external operators have spin and the exchanged operator is not in the symmetric traceless representation of $\mathrm{SO}(d)$ there is no systematic way to determine the blocks. ${ }^{2}$

The paper is divided into two parts. The main goal is to explain how to generalize the series expansion in the radial coordinate introduced by [42] for any bosonic conformal

\footnotetext{
${ }^{1}$ In two dimensions, the CBs associated to the global conformal group are known in closed form [37].

${ }^{2}$ Results in this direction can be obtained using the shadow formalism. In particular, [40, 41] considered the CBs for two external scalars and two vectors. However this method requires further systematization in order to address more general cases.
} 
block in general dimension. This gives rise to a simple way to build CBs in a form which is suitable to the numerical bootstrap approach. Moreover it unravels their connection with the structure of the exchanged conformal representations. In the text, we restrict our attention to external operators in the symmetric traceless representation of $\mathrm{SO}(d)$ but this can be easily generalized using the methods of [40]. In section 2 we review the result of [42] for the scalar CB (we actually consider the slightly more general case of scalar operators of different dimension). As a new result, we explain a strategy to obtain a closed form recurrence relation for the coefficients of the expansion. In section 3 we generalize this technology in order to deal with external operators with spin. We then exemplify the method in three cases: one external vector and three scalars, two external vectors and two scalars and two spin 2 operators and two scalars. Moreover, we explain how to obtain recurrence relations for the coefficients of the expansions in general cases, and how to simplify these recurrence relation in order to make them more efficient (see also appendix A on this point). For clarity of exposition we give examples of simplified closed form recurrence relations only for the two cases of one external vector and three scalars and two external vectors and two scalars. For concreteness we also added to the arXiv submission some Mathematica files which compute the blocks using the recurrence relations for the coefficients.

In section 4 we use an alternative method to find a radial expansion for CBs, by reconstructing their analytic structure as a function of the conformal dimension of the exchanged operator. This method is based on an idea of Zamolodchikov for two dimensional CFTs [43]. It was recently developed in higher dimensions for the scalar conformal block $[13,18]$ and for two external fermions and two scalars in three dimensions [35]. In [44] there was a first attempt to generalize this idea to general external bosonic operators in any dimension. However there was still no explicit computation of CBs for the exchange of a mixed symmetry operator. In section 4 , and also in appendix $\mathrm{B}$, we compute the CBs for two external vectors and two scalars for the exchange of both the symmetric and traceless representation, and the mixed symmetry representation $(l, 1)=\square^{\mid \perp} \square \square$. Again we submitted a Mathematica file which computes the blocks with this method. On one hand this result can be used to match the radial expansion of section 3. More importantly it is a concrete example that shows that the technology of [44] can actually be used to generate in principle any bosonic conformal block.

Finally we conclude the paper with some comments on the possible strategies to obtain more general CBs. We also added appendix $\mathrm{C}$ to explain how the idea of generating some (not all) CBs for external operators with spin by acting with derivatives on the scalar seed block [38] can be implemented in the radial coordinates of [42].

\section{Review of the scalar conformal block}

Let us start by introducing a four point function of scalar operators written in the embedding space. We can expand it in conformal blocks as follows

$$
\left\langle\mathcal{O}_{1}\left(P_{1}\right) \mathcal{O}_{2}\left(P_{2}\right) \mathcal{O}_{3}\left(P_{3}\right) \mathcal{O}_{4}\left(P_{4}\right)\right\rangle=\sum_{\mathcal{O}} c_{12 \mathcal{O}} c_{34 \mathcal{O}} G_{\Delta, l}\left(P_{i}\right),
$$




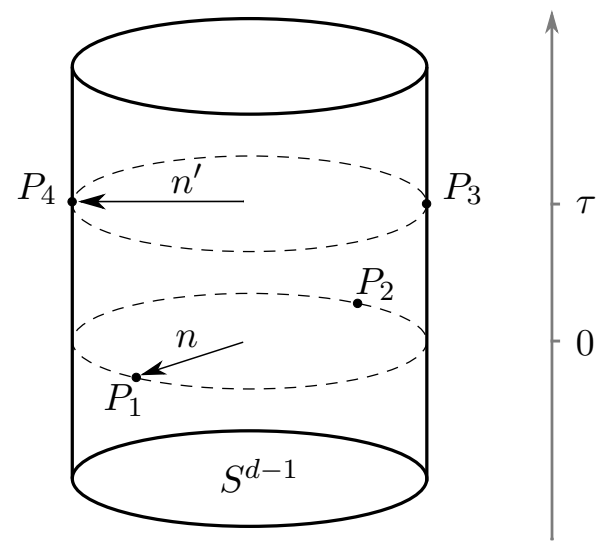

Figure 1. Cylinder configuration that leads to the radial coordinates of [42].

where the sum is over primary operators $\mathcal{O}$ with dimension $\Delta$ and spin $l$. In the embedding space formalism the points are represented by null rays

$$
P=\left(P^{0}, P^{\mu}, P^{d+1}\right) \in \mathbb{R}^{1, d+1}, \quad P^{2}=-\left(P^{0}\right)^{2}+\left(P^{d+1}\right)^{2}+\delta_{\mu \nu} P^{\mu} P^{\nu}=0 .
$$

We will focus on the section $\delta_{\mu \nu} P^{\mu} P^{\nu}=1$. This is naturally parametrized by an Euclidean time $\tau \in \mathbb{R}$ and a unit vector $n^{\mu} \in S^{d-1} \subset \mathbb{R}^{d}$,

$$
P=\left(\cosh \tau, n^{\mu}, \sinh \tau\right) .
$$

The induced metric on this section is just the metric on the cylinder $\mathbb{R} \times S^{d-1}$, with line element $d s^{2}=d \tau^{2}+d \Omega_{S^{d-1}}^{2}$. The following choice of configuration leads to the radial coordinates of $[42]$,

$$
\begin{array}{ll}
P_{1}=(1, n, 0), & P_{3}=\left(\cosh \tau,-n^{\prime}, \sinh \tau\right), \\
P_{2}=(1,-n, 0), & P_{4}=\left(\cosh \tau, n^{\prime}, \sinh \tau\right),
\end{array}
$$

with $n$ and $n^{\prime}$ unit vectors in $\mathbb{R}^{d}$. This choice of null vectors in the embedding space corresponds to the configuration on the cylinder $\mathbb{R} \times S^{d-1}$ shown in figure 1 .

Substituting the choice (2.4) for $P_{i}$ in $G_{\Delta, l}$ we obtain the conformal block on the cylinder,

$$
c_{12 \mathcal{O}} c_{34 \mathcal{O}} G_{\Delta, l}\left(P_{i}\right) \rightarrow \mathcal{G}_{\Delta, l}(r, \eta) .
$$

where we introduced the variables $r$ and $\eta$ of [42],

$$
r=e^{-\tau}, \quad \eta=n \cdot n^{\prime} .
$$

It is trivial to check that $r$ and $\eta$ are related to the usual cross ratios as follows

$$
u=\frac{P_{12} P_{34}}{P_{13} P_{24}}=\frac{16 r^{2}}{\left(1+2 \eta r+r^{2}\right)^{2}}, \quad v=\frac{P_{14} P_{23}}{P_{13} P_{24}}=\frac{\left(1-2 \eta r+r^{2}\right)^{2}}{\left(1+2 \eta r+r^{2}\right)^{2}},
$$

where $P_{i j}=-2 P_{i} \cdot P_{j}$. 


\subsection{A natural series expansion}

On the cylinder it is also possible to write

$$
\mathcal{G}_{\Delta, l}(r, \eta)=\left\langle\mathcal{O}_{4}\left(n^{\prime}\right) \mathcal{O}_{3}\left(-n^{\prime}\right)\left|r^{H_{\mathrm{cyl}}} \mathcal{P}_{l}\right| \mathcal{O}_{2}(-n) \mathcal{O}_{1}(n)\right\rangle,
$$

where $H_{\text {cyl }}$ is the Hamiltonian on the cylinder and $\mathcal{P}_{l}$ is the projector into the conformal family with primary $\mathcal{O}_{\Delta, l}$. It is natural to rewrite the projector as a sum over a complete set of states

$$
\begin{array}{r}
\mathcal{G}_{\Delta, l}(r, \eta)=\sum_{m=0}^{\infty} r^{\Delta+m} \sum_{j=\max (0, l-m)}^{l+m} \sum_{a}\left\langle\mathcal{O}_{4}\left(n^{\prime}\right) \mathcal{O}_{3}\left(-n^{\prime}\right) \mid m, \underline{\mu_{1}\left|\mu_{2}\right| \ldots \mid \mu_{j}}, a\right\rangle \\
\left\langle m, \underline{\mu_{1}\left|\mu_{2}\right| \ldots\left|\mu_{j}\right|}, a \mid \mathcal{O}_{2}(-n) \mathcal{O}_{1}(n)\right\rangle,
\end{array}
$$

where the state $\left|m, \mu_{1}\right| \mu_{2}|\ldots| \mu_{j}|, a\rangle$ represents descendants of the operator $\mathcal{O}$ at the level $m$ and with spin $j$. The label $a$ distinguishes states at the same level and with the same spin. In this case, the only states that contribute are symmetric traceless representations of $\mathrm{SO}(d)$ because the inner products in (2.9) vanish for more general representations. The inner product can be written as

$$
\left\langle m, \underline{\mu_{1}\left|\mu_{2}\right| \ldots \mid \mu_{j}}, a \mid \mathcal{O}_{2}(-n) \mathcal{O}_{1}(n)\right\rangle=u(m, j, a) n_{\left(\mu_{1}\right.} \cdots n_{\left.\mu_{l}\right)},
$$

where the parenthesis select only the traceless and symmetric part of the tensor. We may choose to set $u(0, l)=c_{12 \mathcal{O}} \cdot{ }^{3}$ From these definitions we automatically get

$$
\mathcal{G}_{\Delta, l}(r, \eta)=\sum_{m=0}^{\infty} r^{\Delta+m} \sum_{j=\max (0, l-m)}^{l+m} w(m, j) \mathcal{C}_{j}\left(n \cdot n^{\prime}\right),
$$

where $w(m, j) \equiv \sum_{a} u(m, j, a) \widetilde{u}(m, j, a)$, and $\widetilde{u}(m, j, a)$ comes from the inner product involving operators $\mathcal{O}_{3}$ and $\mathcal{O}_{4}$. The function $\mathcal{C}_{j}$ is a scalar spherical harmonic defined by

$$
\left.\mathcal{C}_{j}\left(n \cdot n^{\prime}\right)=n_{\alpha_{1}} \ldots n_{\alpha_{j}} \pi\left(\alpha_{1}\left|\alpha_{2}\right| \ldots \mid \alpha_{j}\right], \quad \beta_{1}\left|\beta_{2}\right| \ldots\left|\beta_{j}\right|\right) n_{\beta_{1}}^{\prime} \ldots n_{\beta_{j}}^{\prime},
$$

where $\pi$ is the projector into traceless and symmetric tensors with $j$ indices. Moreover, the harmonic function $\mathcal{C}_{l}(\eta)$ can be explicitly written in terms of Gegenbauer polynomials,

$$
\mathcal{C}_{l}(\eta) \equiv \frac{l !}{(2 h-2)_{l}} C_{l}^{(h-1)}(\eta)
$$

where $h=d / 2$. Notice that the normalization in front of the Gegenbauer polynomial is such that $\mathcal{C}_{l}(1)=1$ as expected from formula (2.12) when $n=n^{\prime}$. The crucial point that makes the expansion (2.11) useful is that the coefficients $w(m, j)$ inherit some properties from the structure the conformal representation exchanged in the conformal block. For example, we automatically get that at level $m=0$ in the expansion there exists a single non-zero coefficient. It corresponds to the exchange of the primary, namely

$$
w(0, j)=c_{12 \mathcal{O}} c_{34 \mathcal{O}} \delta_{j, l} .
$$

\footnotetext{
${ }^{3}$ At level $m=0$ there is no degeneracy, so we can drop the label $a$.
} 


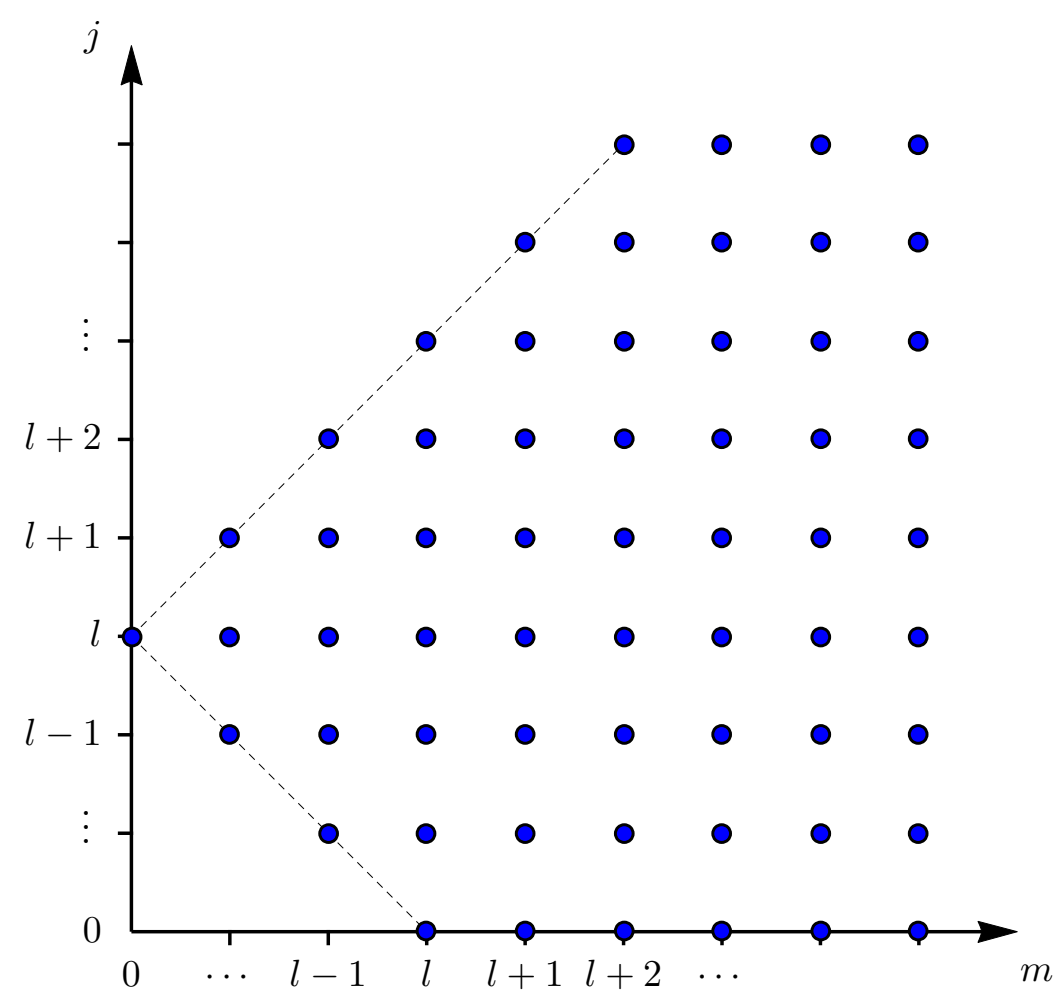

Figure 2. Support of the coefficients $w(m, j)$ in the expansion of $\mathcal{G}_{\Delta, l}$.

Moreover, all level $m$ descendants must have spin $j$ in the interval $[\max (0, l-m), l+m]$, since they are created by the action of $m$ derivatives, as each one of them can at most increase or decrease the spin of the operator by one. Therefore the coefficients have the following structure

$$
w(m, j)=0, \quad|j-l|>m,
$$

which is depicted in figure 2. In addition, if $\mathcal{O}_{4}=\mathcal{O}_{1}$ and $\mathcal{O}_{3}=\mathcal{O}_{2}$ then unitarity implies that all coefficients are non-negative, $w(m, j) \geq 0$.

\subsection{A recurrence relation for the expansion coefficients}

We described the radial expansion for the conformal blocks, but we still need to fix the coefficients $w(m, j)$. In the following we explain how to use the conformal Casimir equation to obtain a recurrence relation for $w(m, j)$. To do so we first exploit the conformal symmetry to fix the conformal partial wave in terms of a function depending only on the cross ratios,

$$
G_{\Delta, l}\left(P_{i}\right)=\frac{\left(\frac{P_{24}}{P_{14}}\right)^{\frac{\Delta_{1}-\Delta_{2}}{2}}\left(\frac{P_{14}}{P_{13}}\right)^{\frac{\Delta_{3}-\Delta_{4}}{2}}}{\left(P_{12}\right)^{\frac{\Delta_{1}+\Delta_{2}}{2}}\left(P_{34}\right)^{\frac{\Delta_{3}+\Delta_{4}}{2}}} g_{\Delta, l}(r, \eta) .
$$

We then write the conformal Casimir equation in the embedding space,

$$
\left(J_{1}+J_{2}\right)^{2} G_{\Delta, l}\left(P_{i}\right)=c_{\Delta, l} G_{\Delta, l}\left(P_{i}\right)
$$


with

$$
c_{\Delta, l}=\Delta(\Delta-2 h)+l(2 h+l-2), \quad J_{k}^{M N}=-i\left(P_{k}^{A} \partial_{P_{k}}^{B}-P_{k}^{B} \partial_{P_{k}}^{A}\right),
$$

in such a way to obtain a second order differential equation for $g_{\Delta, l}(r, \eta)$. To constrain the coefficients $w(m, j)$ we finally need to relate $\mathcal{G}_{\Delta, l}(r, \eta)$ with $g_{\Delta, l}(r, \eta)$. This is trivial, we simply have to write (2.16) using the definitions (2.4) for the points $P_{i}$ on the cylinder,

$$
\mathcal{G}_{\Delta, l}(r, \eta)=c_{12 \mathcal{O}} c_{34 \mathcal{O}} \mathcal{P}(r, \eta) g_{\Delta, l}(r, \eta),
$$

where

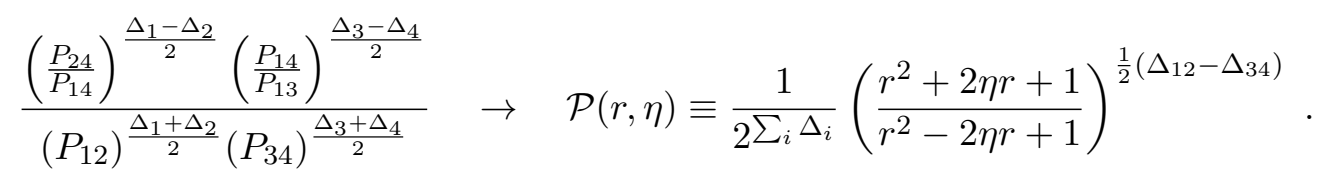

Using these definitions we obtain a differential equation on $\mathcal{G}_{\Delta, l}$,

$$
\begin{aligned}
& \left(r^{2}-1\right)\left[f_{1}^{2} f_{2}^{2} c_{\Delta, l}-4 r\left(\left(\Delta_{12}^{2}+\Delta_{34}^{2}\right) f_{3}+\Delta_{12} \Delta_{34} f_{4} \eta\left(r^{2}+1\right)\right)\right] \mathcal{G}_{\Delta, l} \\
& \quad+f_{1} f_{2} \eta\left(r^{2}-1\right)\left(f_{4}+8 \eta^{2} h r^{2}-2 h\left(r^{2}+1\right)^{2}\right) \partial_{\eta} \mathcal{G}_{\Delta, l}-f_{1}^{2} f_{2}^{2}\left(\eta^{2}-1\right)\left(r^{2}-1\right) \partial_{\eta}^{2} \mathcal{G}_{\Delta, l} \\
& \quad-f_{1} f_{2} r\left[4 \eta^{2} r^{2}\left(-2 h\left(r^{2}+1\right)+r^{2}+3\right)+\left(r^{2}+1\right)\left(2 h\left(r^{2}+1\right)^{2}+r^{4}-8 r^{2}-1\right)\right] \partial_{r} \mathcal{G}_{\Delta, l} \\
& \quad-f_{1}^{2} f_{2}^{2} r^{2}\left(r^{2}-1\right) \partial_{r}^{2} \mathcal{G}_{\Delta, l}=0,
\end{aligned}
$$

where

$$
\begin{array}{ll}
f_{1}=\left(1+r^{2}-2 r \eta\right), & f_{2}=\left(1+r^{2}+2 r \eta\right), \\
f_{3}=r\left(\left(r^{2}+1\right)^{2}-2 \eta^{2}\left(r^{4}+1\right)\right), & f_{4}=r^{2}\left(4 \eta^{2}+r^{2}-6\right)+1 .
\end{array}
$$

We now explain how to convert any Casimir differential equation into closed form recurrence relations for the coefficients of the expansion of the conformal blocks. One can convince oneself that any Casimir equation can be casted in the form ${ }^{4}$

$$
p\left(r, \eta, \partial_{r}, \partial_{\eta}\right) \mathfrak{F}(r, \eta)=0,
$$

where $p\left(x_{1}, x_{2}, x_{3}, x_{4}\right)$ is a polynomial in the variables $x_{i}$ and we choose the order of the variables such that the derivatives are on the right. As an example, (2.21) is of the form (2.24). We want to show that if we expand $\mathfrak{F}(r, \eta)$ as follows

$$
\mathfrak{F}(r, \eta)=\sum_{m, j=0}^{\infty} a_{m, j} \mathfrak{f}_{m, j}(r, \eta), \quad \mathfrak{f}_{m, j}(r, \eta)=r^{m} \mathcal{C}_{j}(\eta),
$$

we are able to recast the differential equation (2.24) as a closed form algebraic relation on the coefficients $a_{m, j}$. To do so we need to know the action of $\eta, \partial_{\eta}, r$ and $\partial_{r}$ on the basis

\footnotetext{
${ }^{4}$ Notice that the Casimir equations may contain rational functions of $r$ and $\eta$. This is not a problem since one can always multiply the full expression by all the denominators.
} 
$\mathfrak{f}_{m, j}(r, \eta)$, that is

$$
\begin{aligned}
r \mathfrak{f}_{m, l}(r, \eta) & =\mathfrak{f}_{m+1, l}(r, \eta), \quad \partial_{r} \mathfrak{f}_{m, l}(r, \eta)=m \mathfrak{f}_{m-1, l}(r, \eta), \\
\eta \mathfrak{f}_{m, l}(r, \eta) & =\frac{1}{2(h+l-1)}\left(l \mathfrak{f}_{m, l-1}(r, \eta)+(2 h+l-2) \mathfrak{f}_{m, l+1}(r, \eta)\right), \\
\partial_{\eta} \mathfrak{f}_{m, l}(r, \eta) & =\frac{1}{\left(1-\eta^{2}\right)} \frac{l(2 h+l-2)}{2(h+l-1)}\left(\mathfrak{f}_{m, l-1}(r, \eta)-\mathfrak{f}_{m, l+1}(r, \eta)\right) .
\end{aligned}
$$

Using these formulas recursively one can obtain the action of any polynomial $p\left(r, \eta, \partial_{r}, \partial_{\eta}\right)$ on $\mathfrak{f}_{m, l}$. The only subtlety comes from the derivatives in $\eta$, since they bring a factor $\left(1-\eta^{2}\right)$ in the denominator. This is not a problem since we can multiply the full equation by $\left(1-\eta^{2}\right)$ each time we use the last equation of (2.26). This is enough to show that we can rewrite (2.24) as a closed form algebraic relation for the coefficients $a_{m, j}$. Nevertheless, in appendix A we explain useful manipulations to simplify the algebraic relations.

Applying this technology to the differential equation (2.21) we obtain that a linear combination of a finite number of coefficients $w(m, j)$ with some shifts in $m$ and $j$ is zero, schematically

$$
\sum_{(\widehat{m}, \widehat{j}) \in \mathcal{S}} c(\widehat{m}, \widehat{j}) w(m+\widehat{m}, j+\widehat{j})=0 .
$$

The set $\mathcal{S}$ that we obtain contains 30 points for example $(0,0),(-1,1),(-1,-1), \ldots$, as pictured in figure 3 , and $c(\widehat{m}, \widehat{j})$ are known functions of the variables $\Delta_{12}, \Delta_{34}, \Delta, l, h$, and $m$ and $j$. We can therefore express the coefficient $w(m, j)$ with maximal $m$ (the point at the origin of the axes in figure 3) as a linear combination of $w\left(m^{\prime}, j^{\prime}\right)$ with lower $m^{\prime}$.

Moreover, if we set $m=0$ in (2.27) we obtain a constraint on the possible initial conditions

$$
(j-l) w(0, j)=0,
$$

which is solved imposing $w(0, j)=0$ for $j \neq l$. For $j=l$, instead, the coefficient $w(0, l)$ is left unrestricted. This is consistent with the initial condition (2.14) obtained from the structure of the conformal families.

Notice also that the restriction on the initial condition, together with the shape of figure 3 , imply that the coefficients of the expansion organize as pictured in figure 2 , which was obtained from the properties of conformal families.

Equation (2.27) with the initial condition (2.14) is enough to determine recursively the coefficients $w(m, j)$ for any $m$ and $j$. We will not present here explicitly the relation (2.27) since the formula is very long. Instead, it is defined in a Mathematica file. As an example we present the first coefficients of the expansion for generic parameters

$$
\begin{aligned}
& w(1, l-1)=\frac{\Delta_{12} \Delta_{34} l}{(h+l-1)(-\Delta+2 h+l-2)}, \\
& w(1, l+1)=-\frac{\Delta_{12} \Delta_{34}(2 h+l-2)}{(h+l-1)(\Delta+l)} .
\end{aligned}
$$

Notice that they go to zero when $\Delta_{34}=0=\Delta_{12}$, as expected from the results of [42]. 


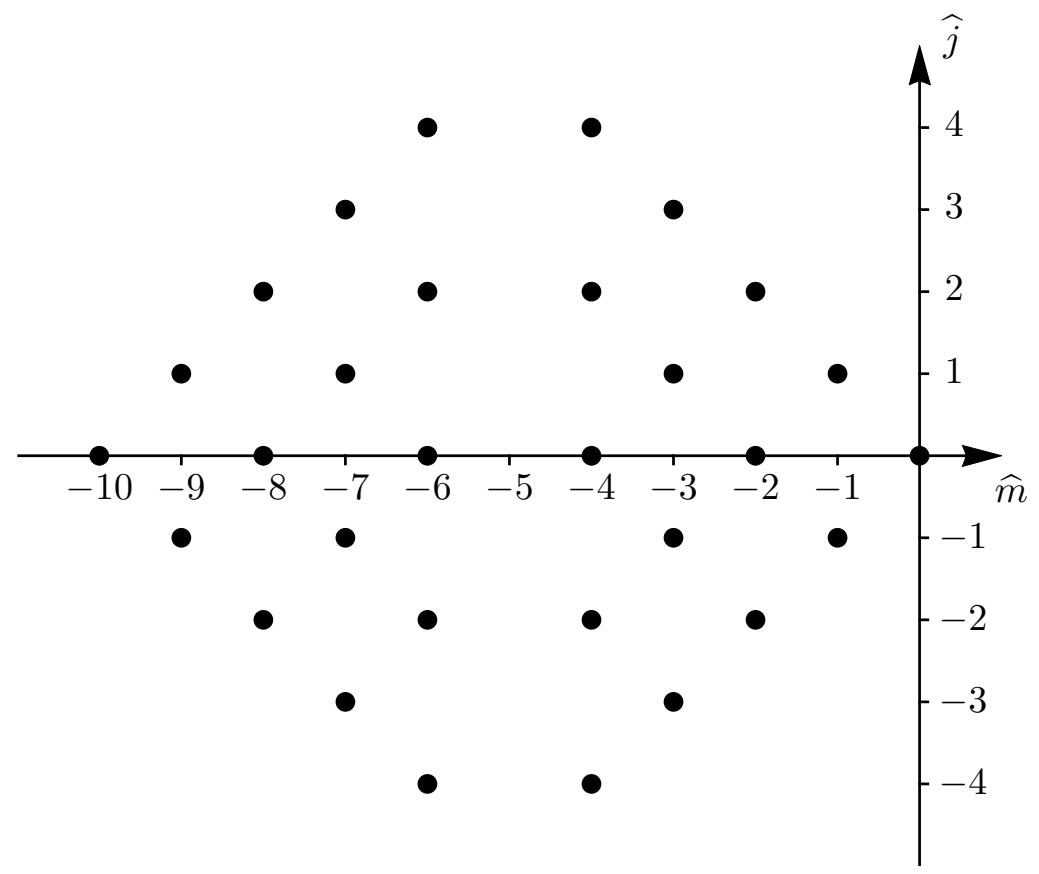

Figure 3. The set of points $\mathcal{S}$ involved in the recurrence relation for the coefficients $w(m, j)$ of the scalar conformal block.

Formula (2.27) is a new result, since in [42] it was not obtained a closed form recurrence relation for the coefficients of the expansion. The main reason for this is that the authors of [42] kept some rational functions of $r$ and $\eta$ (which have infinite expansion in small $r$ ) in the Casimir differential equation. Thus the associated recurrence relation would have had a set $\mathcal{S}$ containing an infinite number of points. On the other hand, multiplying the Casimir equation by all the denominators it becomes possible to find a recurrence relation with a finite set $\mathcal{S}$. Notice that this example explicitly shows that there exist many possible equivalent recurrence relations, which however are not equivalently efficient. In fact we expand on this point in appendix A, where we explain some strategies to further reduce the number of points in $\mathcal{S}$ with respect to the algorithm proposed in the main text. We would like to stress that while some features of the figure 3 have a physical meaning (they are related to the structure of conformal families as mentioned above), some others come just as a result of the computation and we do not know why they should hold from general principles. For example it is not clear why a priori we should expect a symmetric picture (with respect to the axis $\widehat{m}=-5$ ) and why the points at $\widehat{m}=-5$ are absent. In fact it would be interesting to understand which features are necessary and which is the minimal set $\mathcal{S}$.

As a last comment we mention that the recurrence relation (2.27) can further simplify when we specialize the value of some parameters. For example when $\Delta_{12}=0=\Delta_{34}$ all the coefficients $c(\widehat{m}, \widehat{j})$ for $\widehat{m}$ odd vanish, therefore the actual support $\mathcal{S}$ reduces to a set of 18 points. Since the recurrence relation is written in an analytic form it is also possible to access limit cases when some parameters (like $\Delta, l, h)$ are large. For more details about 
the simplification of the recurrence relation in special cases, the reader may check the Mathematica files where all the definitions are presented explicitly.

\section{Spinning conformal blocks}

In this section we present the general framework to obtain the radial expansion of conformal blocks for external bosonic operators with spin. Initially we exemplify the formalism applying it to the simpler case of one external vector operator and three scalars, for which the allowed exchanged operators are still symmetric and traceless. After that, in separate subsections, we apply the method to the cases of two external vectors and two scalars, and of two spin 2 operators and two scalars. In these cases there are new mixed tensor operators that can be exchanged.

\subsection{General formalism and example of one vector and three scalars}

We first introduce the conformal block expansion in the embedding formalism of a four point function of operators $\mathcal{O}_{i}$ with spin $l_{i}$ and conformal dimension $\Delta_{i}$

$$
\left\langle\mathcal{O}_{1}\left(P_{1}, Z_{1}\right) \mathcal{O}_{2}\left(P_{2}, Z_{2}\right) \mathcal{O}_{3}\left(P_{3}, Z_{3}\right) \mathcal{O}_{4}\left(P_{4}, Z_{4}\right)\right\rangle=\sum_{\mathcal{O}} \sum_{p, q} c_{12 \mathcal{O}}^{(p)} c_{34 \mathcal{O}}^{(q)} G_{\lambda}^{(p, q)}\left(P_{i}, Z_{i}\right)
$$

where the first sum runs over all the primary operators $\mathcal{O}$ in representations labeled by $\lambda=\left(\Delta, l_{1}, l_{2}, \ldots, l_{\left[\frac{d}{2}\right]}\right)$, where $[x]$ rounds $x$ down to the closest integer. We also sum over $p$ and $q$, which label the possible conformal invariant tensor structures of the three point functions $\left\langle\mathcal{O}_{1} \mathcal{O}_{2} \mathcal{O}\right\rangle$ and $\left\langle\mathcal{O}_{3} \mathcal{O}_{4} \mathcal{O}\right\rangle$, respectively. Let us recall that the auxiliary variables $Z_{i}$ are a convenient way to avoid dealing with tensor indices in embedding space, that is $\mathcal{O}(P, Z)=Z^{A_{1}} \ldots Z^{A_{l}} \mathcal{O}_{A_{1} \ldots A_{l}}(P)$. As an example, for one external vector we have

$$
\left\langle\mathcal{O}_{1}\left(P_{1}, Z_{1}\right) \mathcal{O}_{2}\left(P_{2}\right) \mathcal{O}_{3}\left(P_{3}\right) \mathcal{O}_{4}\left(P_{4}\right)\right\rangle=\sum_{\mathcal{O}} \sum_{p=1}^{2} c_{12 \mathcal{O}}^{(p)} c_{34 \mathcal{O}} G_{\Delta, l}^{(p)}\left(P_{i}, Z_{1}\right) .
$$

The two possible values $p=1,2$ correspond to the two possible tensor structures of the three-point function $\left\langle\mathcal{O}_{1}\left(P_{1}, Z_{1}\right) \mathcal{O}_{2}\left(P_{2}\right) \mathcal{O}(P, Z)\right\rangle$ between external operators $\mathcal{O}_{1}, \mathcal{O}_{2}$ and the exchanged operator $\mathcal{O}$, which in this example can only be symmetric traceless of generic spin $l$. As in the scalar case, we are interested in writing the conformal blocks in the cylinder configuration. To do so we can use formulae (2.4) and the following definition for the embedding space polarization vectors

$$
\begin{array}{ll}
Z_{1}=\left(z_{1} \cdot n, z_{1}, z_{1} \cdot n\right), & Z_{3}=\left(-z_{3} \cdot n^{\prime} e^{\tau}, z_{3},-z_{3} \cdot n^{\prime} e^{\tau}\right), \\
Z_{2}=\left(-z_{2} \cdot n, z_{2},-z_{2} \cdot n\right), & Z_{4}=\left(z_{4} \cdot n^{\prime} e^{\tau}, z_{4}, z_{4} \cdot n^{\prime} e^{\tau}\right),
\end{array}
$$

where $z_{i}$ are polarization vectors in $\mathbb{R}^{d}$ which obey $z_{i}^{2}=0$. Notice that $Z_{i}^{2}=Z_{i} \cdot P_{i}=0$, as it should [45]. With these definitions we get

$$
\sum_{p, q} c_{12 \mathcal{O}}^{(p)} c_{34 \mathcal{O}}^{(q)} G_{\lambda}^{(p, q)}\left(P_{i}, Z_{i}\right) \rightarrow \mathcal{G}_{\lambda}\left(r, \eta, n \cdot z_{i}, n^{\prime} \cdot z_{i}, z_{i} \cdot z_{j}\right)
$$


Therefore $\mathcal{G}_{\lambda}^{(p, q)}$ can be expanded in polynomials $p_{s}$ of the variables $n \cdot z_{i}, n^{\prime} \cdot z_{i}, z_{i} \cdot z_{j}$ with weight $l_{i}$ in each variable $z_{i}$,

$$
\mathcal{G}_{\lambda}\left(r, \eta, n \cdot z_{i}, n^{\prime} \cdot z_{i}, z_{i} \cdot z_{j}\right)=\sum_{s} p_{s}\left(n \cdot z_{i}, n^{\prime} \cdot z_{i}, z_{i} \cdot z_{j}\right) F_{s}(r, \eta)
$$

where the coefficient multiplying each polynomial is a function $F_{s}$ of $r$, and $\eta$ and $s$ labels the independent tensor structures of the four-point function. For one external vector we have

$$
\mathcal{G}_{\Delta, l}=\left(n \cdot z_{1}\right) F_{1}(r, \eta)+\left(n^{\prime} \cdot z_{1}\right) F_{2}(r, \eta) .
$$

On the other hand, just as for the scalar case (2.8), the conformal block on the cylinder can be written as

$$
\mathcal{G}_{\lambda}\left(r, \eta, n \cdot z_{i}, n^{\prime} \cdot z_{i}, z_{i} \cdot z_{j}\right)=\left\langle\mathcal{O}_{4}\left(n^{\prime}, z_{4}\right) \mathcal{O}_{3}\left(-n^{\prime}, z_{3}\right)\left|r^{H_{\mathrm{cyl}}} \mathcal{P}_{\lambda}\right| \mathcal{O}_{2}\left(-n, z_{2}\right) \mathcal{O}_{1}\left(n, z_{1}\right)\right\rangle,
$$

where $\mathcal{P}_{\lambda}$ is the projector into the conformal family with highest weight $\lambda$. It is natural to rewrite the projector as a sum over a complete basis of states

$$
\mathcal{G}_{\lambda}=\sum_{m=0}^{\infty} r^{\Delta+m} \sum_{\mathbf{Y}} \sum_{a}\left\langle\mathcal{O}_{4}\left(n^{\prime}, z_{4}\right) \mathcal{O}_{3}\left(-n^{\prime}, z_{3}\right) \mid m, \mathbf{Y}, a\right\rangle\left\langle m, \mathbf{Y}, a \mid \mathcal{O}_{2}\left(-n, z_{2}\right) \mathcal{O}_{1}\left(n, z_{1}\right)\right\rangle,
$$

where we sum over all states at level $m$ of the conformal family, organized in irreducible representations (irreps) $\mathbf{Y}$ of $\mathrm{SO}(d)$. For example, for one external vector, the representation $\mathbf{Y}$ can only be symmetric and traceless, therefore

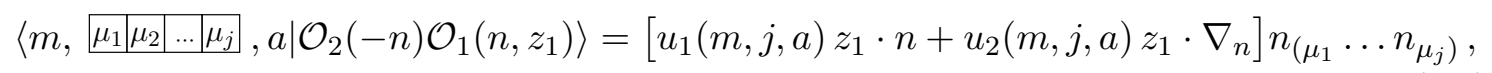

where

$$
\left(\nabla_{n}\right)_{\mu}=\frac{\partial}{\partial n^{\mu}}-n_{\mu} n \cdot \frac{\partial}{\partial n}
$$

computes the covariant derivative on the sphere $S^{d-1}$ parametrized by $n$ and the coefficients $u_{k}$ are not fixed by rotational invariance. We can choose a basis for the OPE coefficients $c_{12 \mathcal{O}}^{(p)}$ such that

$$
\left\langle 0, \underline{\mu_{1}\left|\mu_{2}\right| \ldots \mid \mu_{j}} \mid \mathcal{O}_{2}(-n) \mathcal{O}_{1}\left(n, z_{1}\right)\right\rangle=\left[c_{12 \mathcal{O}}^{(1)} z_{1} \cdot n+c_{12 \mathcal{O}}^{(2)} z_{1} \cdot \nabla_{n}\right] n_{\left(\mu_{1}\right.} \ldots n_{\left.\mu_{j}\right)} .
$$

In this formalism we can easily deal with more complicated irreps $\mathbf{Y}$ of $\mathrm{SO}(d)$, which will appear in the next sections. For now, we continue the study of the case with one external vector, for which (3.8) becomes

$$
\mathcal{G}_{\Delta, l}=\sum_{m=0}^{\infty} r^{\Delta+m} \sum_{j} \sum_{a} \widetilde{u}(m, j, a)\left[u_{1}(m, j, a) z_{1} \cdot n+u_{2}(m, j, a) z_{1} \cdot \nabla_{n}\right] \mathcal{C}_{j}\left(n \cdot n^{\prime}\right),
$$

where $\widetilde{u}(m, j, a)$ comes from the inner product of the scalars $\mathcal{O}_{3}$ and $\mathcal{O}_{4}$ with the exchanged states. It is convenient to introduce the following two functions of $r$ and $\eta$,

$$
W_{s}(r, \eta) \equiv \sum_{m=0}^{\infty} r^{\Delta+m} \sum_{j=\max (0, l-m)}^{l+m} w_{s}(m, j) \mathcal{C}_{j}(\eta), \quad s=1,2,
$$


where $w_{s}(m, j) \equiv \sum_{a} \widetilde{u}(m, j, a) u_{s}(m, j, a)$. The choice (3.11) corresponds to the following initial condition

$$
w_{s}(0, j)=c_{12 \mathcal{O}}^{(s)} c_{34 \mathcal{O}} \delta_{j, l} .
$$

The functions $W_{s}$ are the basic building blocks for the radial expansion. However they are related in a non trivial way to the full partial wave $G_{\lambda}$ in (3.1). In the following we explain how to relate these functions in a generic framework while always exemplifying it for the case of one external vector operator.

As a first step we relate the functions $W_{s}$ to the functions $F_{s}$ defined in (3.5). For one external vector this can be done expanding (3.12) and collecting terms according to (3.6),

$$
F_{1}(r, \eta)=W_{1}(r, \eta)-\eta \partial_{\eta} W_{2}(r, \eta), \quad F_{2}(r, \eta)=\partial_{\eta} W_{2}(r, \eta) .
$$

The second step is to relate the conformal partial waves $G_{\lambda}$ to the functions $F_{s}$. To do so we exploit conformal symmetry to write the full partial wave in terms of functions of the cross ratios. This can be easily done for any conformal block in the embedding formalism,

$$
G_{\lambda}^{(p, q)}\left(P_{i}, Z_{i}\right)=\frac{\left(\frac{P_{24}}{P_{14}}\right)^{\frac{\Delta_{1}-\Delta_{2}}{2}}\left(\frac{P_{14}}{P_{13}}\right)^{\frac{\Delta_{3}-\Delta_{4}}{2}}}{\left(P_{12}\right)^{\frac{\Delta_{1}+\Delta_{2}}{2}}\left(P_{34}\right)^{\frac{\Delta_{3}+\Delta_{4}}{2}}} \sum_{s} g_{\lambda, s}^{(p, q)}(r, \eta) Q^{(s)}\left(P_{i}, Z_{i}\right),
$$

where the set of $Q^{(s)}\left(P_{i}, Z_{i}\right)$ encode the conformal invariant tensor structures and can be generated using $[40,45]$

$$
\begin{aligned}
V_{i, j k} & \equiv-\frac{\left(P_{i} \cdot P_{j}\right)\left(P_{k} \cdot Z_{i}\right)-\left(P_{i} \cdot P_{k}\right)\left(P_{j} \cdot Z_{i}\right)}{\sqrt{-2\left(P_{i} \cdot P_{j}\right)\left(P_{i} \cdot P_{k}\right)\left(P_{j} \cdot P_{k}\right)}}, \\
H_{i j} & \equiv \frac{\left(P_{i} \cdot P_{j}\right)\left(Z_{i} \cdot Z_{j}\right)-\left(P_{i} \cdot Z_{j}\right)\left(P_{j} \cdot Z_{i}\right)}{\left(P_{i} \cdot P_{j}\right)} .
\end{aligned}
$$

Finally we map (3.16) to the cylinder using the definitions (2.4) and (3.3), and we collect terms according to (3.5). In this way we obtain a linear relation between the functions $g_{\lambda, s}$ and $F_{s}$.

In the one vector case we only have two possible tensor structures in (3.16),

$$
Q^{(1)}\left(P_{i}, Z_{i}\right)=V_{1,23}, \quad Q^{(2)}\left(P_{i}, Z_{i}\right)=V_{1,43},
$$

which can be evaluated on the cylinder using (2.4) and (3.3),

$$
\begin{aligned}
& V_{1,23} \rightarrow \frac{\left(-r^{2}+2 \eta r+1\right) n \cdot z_{1}-2 r z_{1} \cdot n^{\prime}}{\left(r^{2}-2 \eta r+1\right)^{1 / 2}\left(r^{2}+2 \eta r+1\right)^{1 / 2}}, \\
& V_{1,43} \rightarrow \frac{2 \eta r^{2} n \cdot z_{1}-\left(r^{2}+1\right) z_{1} \cdot n^{\prime}}{\left(r^{2}-2 \eta r+1\right)^{1 / 2}\left(r^{2}+2 \eta r+1\right)^{1 / 2}} .
\end{aligned}
$$

We then replace (3.19) and (2.20) in (3.16). Collecting terms according to (3.6) we finally get

$$
\begin{aligned}
& \sum_{p=1}^{2} c_{12 \mathcal{O}}^{(p)} c_{34 \mathcal{O}} g_{1}^{(p)}(r, \eta)=\mathcal{A}(r, \eta)\left[2 \eta r^{2} F_{2}(r, \eta)+\left(r^{2}+1\right) F_{1}(r, \eta)\right] \\
& \sum_{p=1}^{2} c_{12 \mathcal{O}}^{(p)} c_{34 \mathcal{O}} g_{2}^{(p)}(r, \eta)=\mathcal{A}(r, \eta)\left[\left(r^{2}-2 \eta r-1\right) F_{2}(r, \eta)-2 r F_{1}(r, \eta)\right]
\end{aligned}
$$




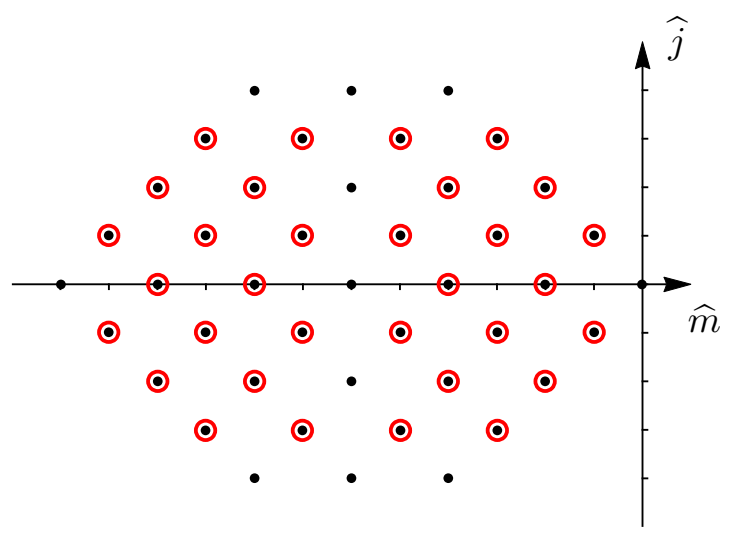

(a) $s=1$

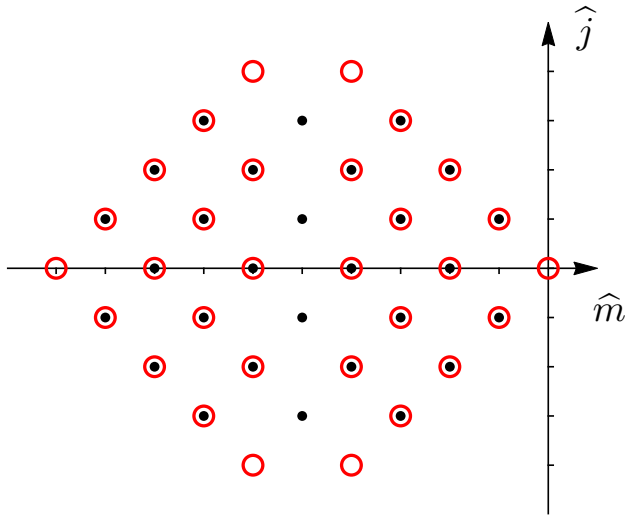

(b) $s=2$

Figure 4. The sets $\mathcal{S}_{s^{\prime}}^{s}$ appearing in the linear combination (3.24) for $s=1,2$. Red circles correspond to $s^{\prime}=2$ and black dots correspond to $s^{\prime}=1$.

where we defined the function

$$
\mathcal{A}(r, \eta)=-\frac{2^{\sum_{i} \Delta_{i}}}{1-r^{2}}\left(\frac{1-2 \eta r+r^{2}}{1+2 \eta r+r^{2}}\right)^{\frac{1}{2}\left(\Delta_{12}-\Delta_{34}+1\right)} .
$$

With the above relations we can write $G_{\lambda}$ in terms of the functions $W_{s}$. The last step is to find a recurrence relation for the coefficients $w_{s}(m, j)$ of the functions $W_{s}$. To do so, we first write the conformal Casimir equation in the embedding space,

$$
\left(J_{1}+J_{2}\right)^{2} G_{\lambda}\left(P_{i}, Z_{i}\right)=c_{\lambda} G_{\lambda}\left(P_{i}, Z_{i}\right), \quad c_{\lambda}=\Delta(\Delta-2 h)+\sum_{i=1}^{[h]} l_{i}\left(l_{i}+2 h-2 i\right),
$$

where $J_{i}$ are the generators of conformal transformations

$$
J_{k}^{M N}=-i\left(P_{k}^{M} \partial_{P_{k}}^{N}-P_{k}^{N} \partial_{P_{k}}^{M}+Z_{k}^{M} \partial_{Z_{k}}^{N}-Z_{k}^{N} \partial_{Z_{k}}^{M}\right)
$$

We then write $G_{\lambda}$ in terms of $W_{s}$, which leads to coupled differential equations for the functions $W_{s}$. Repeating the same procedure described in the scalar case, we can trade (see also appendix A) the differential equations for coupled recurrence relations on the coefficients $w_{s}(m, j)$. When we do this for the case of one external vector we obtain two coupled recurrence relations of the kind (the precise formulae are defined in a Mathematica file)

$$
\sum_{s^{\prime}=1}^{2} \sum_{(\widehat{m}, \widehat{j}) \in \mathcal{S}_{s^{\prime}}^{s}} c_{s^{\prime}}^{s}(\widehat{m}, \widehat{j}) w_{s^{\prime}}(m+\widehat{m}, j+\widehat{j})=0, \quad s=1,2,
$$

where the sets of point $\mathcal{S}_{s^{\prime}}^{s}$ are represented in figures $4(\mathrm{a})$ and $4(\mathrm{~b})$. The sets $\mathcal{S}_{1}^{s}$ are pictured by black dots while the set $\mathcal{S}_{2}^{s}$ by red circles. In particular the sets $\mathcal{S}_{s^{\prime}}^{1}$ of the first recurrence relation are shown in figure $4\left(\right.$ a) while the sets $\mathcal{S}_{s^{\prime}}^{2}$ of the second one are in 
figure 4(b). We can then obtain the coefficients of the expansion iterating (3.24) with the initial condition (3.14). Equation (3.24) for $m=0$ reduces to

$$
(j-l) w_{1}(0, j)=0, \quad j(j-l) w_{2}(0, j)=0,
$$

which constraints the possible initial conditions. In fact, (3.25) implies $w_{1}(0, j)=$ $w_{2}(0, j)=0$, for any $j \neq l$. On the other hand, $w_{1}(0, l)$ and $w_{2}(0, l)$ are not constrained by equation (3.24). This is consistent with the initial condition (3.14) that we derived from the structure of the conformal families. The two independent solutions of equation (3.24) are the two conformal blocks that we labeled with $p=1,2$. The last remark is that (3.24) does not constrain $w_{2}(m, 0)$. This actually make sense since in (3.15) the function $W_{2}$ only appears derived once in $\eta$ and therefore the $j=0$ term is annihilated. In fact, for practical purposes one could redefine $W_{2}$ avoiding to sum over the terms with $j=0$.

As an example we present all the coefficients at level $m=1$ of the expansion of the functions $W_{s}$, which build the conformal block $G_{\Delta, l}^{(p)}$. At level zero we set the initial condition $w_{s}(0, l)=\delta_{s, p}$. Using (3.24) we therefore obtain

$$
\begin{aligned}
& w_{1}(1, l+1)=-\frac{\Delta_{34}(2 h+l-2)\left(\Delta_{12} \delta_{p, 1}+l \delta_{p, 2}\right)}{(h+l-1)(\Delta+l)}, \\
& w_{2}(1, l+1)=-\frac{\Delta_{34}(2 h+l-2)\left(\delta_{p, 1}+\Delta_{12} l \delta_{p, 2}\right)}{(l+1)(h+l-1)(\Delta+l)}, \\
& w_{1}(1, l-1)=\frac{\Delta_{34} l\left(\Delta_{12} \delta_{p, 1}-(2 h+l-2) \delta_{p, 2}\right)}{(h+l-1)(-\Delta+2 h+l-2)}, \\
& w_{2}(1, l-1)=-\frac{\Delta_{34} l\left(\delta_{p, 1}-\Delta_{12}(2 h+l-2) \delta_{p, 2}\right)}{(h+l-1)(2 h+l-3)(-\Delta+2 h+l-2)} .
\end{aligned}
$$

Finally it is worth mentioning that the case $\Delta_{12}=0=\Delta_{34}$ drastically simplifies the recurrence relations. In fact in this case not only all the $c_{s^{\prime}}^{s}(\widehat{m}, \widehat{j})$ with odd $\widehat{m}$ are automatically zero, but also the two recurrence relations completely decouple. In fact $c_{s^{\prime}}^{s}(\widehat{m}, \widehat{j})=0$ if $s^{\prime} \neq s$. We stress that this result comes out by direct computation, but we do not have any insight of why this needs to happen. For more details about the simplification of the recurrence relations for special values of the parameters we encourage the reader to use the Mathematica files.

\subsection{Two external vectors and two scalars}

In this section we describe how to obtain a radial expansion for conformal blocks of two external vectors and two scalars. In particular, we consider the following conformal block decomposition

$$
\begin{aligned}
\left\langle\mathcal{O}_{1}\left(P_{1}, Z_{1}\right) \mathcal{O}_{2}\left(P_{2}\right) \mathcal{O}_{3}\left(P_{3}, Z_{3}\right) \mathcal{O}_{4}\left(P_{4}\right)\right\rangle= & \sum_{\mathcal{O} \in \mathbb{W}} \sum_{p, q=1}^{2} c_{12 \mathcal{O}}^{(p)} c_{34 \mathcal{O}}^{(q)} G_{\Delta, l}^{(p, q)}\left(P_{i}, Z_{i}\right) \\
& +\sum_{\mathcal{O} \in \Psi^{W}} c_{12 \mathcal{O}} c_{34 \mathcal{O}} G_{\Delta, l, 1}\left(P_{i}, Z_{i}\right)
\end{aligned}
$$

There exist four conformal partial waves $G_{\Delta, l}^{(p, q)}$ (for $\left.p, q=1,2\right)$ when the exchanged multiplet has an highest weight $\mathcal{O}$ in the symmetric and traceless representation. Additionally, 
there is a single partial wave $G_{\Delta, l, 1}$ when $\mathcal{O}$ is in the representation $(\Delta, l, 1)$. We shall see that the radial expansion allows us to find all the cases at once.

First, we consider the contribution from each conformal family on the cylinder

$$
\sum_{p, q=1}^{2} c_{12 \mathcal{O}}^{(p)} c_{34 \mathcal{O}}^{(q)} G_{\Delta, l}^{(p, q)}\left(P_{i}, Z_{i}\right) \rightarrow \mathcal{G}_{\Delta, l}, \quad c_{12 \mathcal{O}} c_{34 \mathcal{O}} G_{\Delta, l, 1}\left(P_{i}, Z_{i}\right) \rightarrow \mathcal{G}_{\Delta, l, 1}
$$

On the cylinder, both these functions can be expanded in the following tensor structures

$$
\begin{aligned}
\mathcal{G}_{\lambda}= & z_{1} \cdot n z_{3} \cdot n^{\prime} F_{1}(r, \eta)+z_{1} \cdot n z_{3} \cdot n F_{2}(r, \eta)+ \\
& +z_{1} \cdot n^{\prime} z_{3} \cdot n^{\prime} F_{3}(r, \eta)+z_{1} \cdot n^{\prime} z_{3} \cdot n F_{4}(r, \eta)+z_{1} \cdot z_{3} F_{5}(r, \eta) .
\end{aligned}
$$

The functions $F_{s}$ depend on the representation $\lambda$ but we omit this label to avoid cluttering the equations.

To implement the expansion (3.8) for two external vectors we need to consider the contribution from two types of states at each level $m$ (the descendants of the two $\mathrm{SO}(d)$ irreps that are exchanged in this case). The first is the same that we obtained in the one external vector case (3.9). The second one is new, with a coupling between one of the vectors and one of the scalars written as

$$
\begin{aligned}
&\left\langle m, \frac{\mu_{1}\left|\mu_{2}\right| \ldots \mid \mu_{j}}{\nu}, a \mid \mathcal{O}_{2}(-n) \mathcal{O}_{1}\left(n, z_{1}\right)\right\rangle \\
& \quad=N_{\mathcal{V}} u_{0}(m, j, a) \pi\left(\frac{\mu_{1}\left|\mu_{2}\right| \ldots \mid \mu_{j}}{\nu}, \frac{\alpha_{1}\left|\alpha_{2}\right| \ldots \mid \alpha_{j}}{\beta \beta}\right)\left(z_{1}\right)_{\beta} n_{\alpha_{1}} \ldots n_{\alpha_{j}}
\end{aligned}
$$

where the normalization constant $N_{\mathcal{V}}$ is introduced for later convenience. Notice that the value at $m=0$ of $u_{s}(0, j)$ has to be different depending on what is the representation $\lambda$ of the exchanged primary operator. In fact, if $\lambda$ is the symmetric and traceless representation, then

$$
u_{0}(0, l)=0, \quad u_{q}(0, l)=c_{12 \mathcal{O}}^{(q)}, \quad q=1,2,
$$

and when $\lambda=(\Delta, l, 1)$, then

$$
u_{0}(0, l)=c_{12 \mathcal{O}}, \quad u_{q}(0, l)=0, \quad q=1,2 .
$$

We are lead to the following expansion for the conformal block on the cylinder

$$
\begin{aligned}
\mathcal{G}_{\lambda}=\sum_{m=0}^{\infty} r^{\Delta+m} \sum_{j} \sum_{a}\left[N_{\mathcal{V}}^{2}\right. & u_{0}(m, j, a) \widetilde{u}_{0}(m, j, a)\left(z_{1}\right)_{\mu}\left(z_{3}\right)_{\nu} \mathcal{V}_{j}^{\mu \nu}\left(n, n^{\prime}\right) \\
& +\left(u_{1}(m, j, a) z_{1} \cdot n+u_{2}(m, j, a) z_{1} \cdot \nabla_{n}\right) \\
& \left.\times\left(\widetilde{u}_{1}(m, j, a) z_{3} \cdot n^{\prime}+\widetilde{u}_{2}(m, j, a) z_{3} \cdot \nabla_{n^{\prime}}\right) \mathcal{C}_{j}\left(n \cdot n^{\prime}\right)\right]
\end{aligned}
$$

where

$$
\mathcal{V}_{j}^{\mu \nu}\left(n, n^{\prime}\right)=n_{\alpha_{1}} \ldots n_{\alpha_{j}} \pi\left(\frac{\alpha_{1}\left|\alpha_{2}\right| \ldots \mid \alpha_{j}}{\mu}, \frac{\beta_{1}\left|\beta_{2}\right| \ldots \mid \beta_{j}}{\nu}\right) n_{\beta_{1}}^{\prime} \ldots n_{\beta_{j}}^{\prime}
$$


is a (double) vector spherical harmonic ${ }^{5}$ with index $\mu$ at the point $n$ and index $\nu$ at the point $n^{\prime}$. The double vector harmonic can be expressed in terms of Gegenbauer functions as follows [46]

$$
\begin{aligned}
N_{\mathcal{V}}^{2} \mathcal{V}_{l}^{\mu \nu}\left(n, n^{\prime}\right)\left(z_{1}\right)_{\mu}\left(z_{3}\right)_{\nu}= & \mathcal{C}_{l}^{\prime \prime}(\eta)\left(-\eta n \cdot z_{1} z_{3} \cdot n^{\prime}+z_{1} \cdot n^{\prime} z_{3} \cdot n^{\prime}+n \cdot z_{1} n \cdot z_{3}-z_{1} \cdot z_{3}\right) \\
& -\left(n \cdot z_{3} z_{1} \cdot n^{\prime}-\eta z_{1} \cdot z_{3}\right)\left((2 h-2) \mathcal{C}_{l}^{\prime}(\eta)+\eta \mathcal{C}_{l}^{\prime \prime}(\eta)\right),
\end{aligned}
$$

where the normalization constant $N_{\mathcal{V}}^{2}$ is fixed by requiring that $\pi$ is a projector

$$
N_{\mathcal{V}}^{2} \equiv \frac{2^{l} l(h)_{l-1}}{(2 h-1)_{l-2}} .
$$

It is then convenient to introduce five functions which have a nice expansion in radial coordinates and which encode the information to generate the conformal blocks

$$
W_{s}(r, \eta)=\sum_{j, m} w_{s}(j, m) r^{\Delta+m} \mathcal{C}_{j}(\eta), \quad s=1,2,3,4,5,
$$

with

$$
\begin{aligned}
& w_{1}(m, j)=\sum_{a} u_{1}(m, j, a) \widetilde{u}_{1}(m, j, a), \\
& w_{2}(m, j)=\sum_{a} u_{1}(m, j, a) \widetilde{u}_{2}(m, j, a), \\
& w_{3}(m, j)=\sum_{a} u_{2}(m, j, a) \widetilde{u}_{1}(m, j, a), \\
& w_{4}(m, j)=\sum_{a} u_{2}(m, j, a) \widetilde{u}_{2}(m, j, a), \\
& w_{5}(m, j)=\sum_{a} u_{0}(m, j, a) \widetilde{u}_{0}(m, j, a) .
\end{aligned}
$$

Next we want to write the conformal partial waves in terms of the functions $W_{s}$. First we obtain the relation between $F_{s}$ and $W_{s}$ according to the definition (3.29),

$$
\begin{aligned}
& F_{1}(r, \eta)=W_{1}(r, \eta)+\eta \partial_{\eta}\left[\eta \partial_{\eta} W_{4}(r, \eta)-W_{2}(r, \eta)-W_{3}(r, \eta)-\partial_{\eta} W_{5}(r, \eta)\right], \\
& F_{2}(r, \eta)=\partial_{\eta}\left[-\eta \partial_{\eta} W_{4}(r, \eta)+W_{2}(r, \eta)+\partial_{\eta} W_{5}(r, \eta)\right] \\
& F_{3}(r, \eta)=\partial_{\eta}\left[-\eta \partial_{\eta} W_{4}(r, \eta)+W_{3}(r, \eta)+\partial_{\eta} W_{5}(r, \eta)\right] \\
& F_{4}(r, \eta)=-\left[2(-1+h)+\eta \partial_{\eta}\right] \partial_{\eta} W_{5}(r, \eta)+\partial_{\eta}{ }^{2} W_{4}(r, \eta), \\
& F_{5}(r, \eta)=\left[2(-1+h) \eta+\left(-1+\eta^{2}\right) \partial_{\eta}\right] \partial_{\eta} W_{5}(r, \eta)+\partial_{\eta} W_{4}(r, \eta) .
\end{aligned}
$$

Secondly we write the conformal partial wave in the embedding space in terms of functions of the cross ratios

$$
\sum_{p, q} c_{12 \mathcal{O}}^{(p)} c_{34 \mathcal{O}}^{(q)} G_{\lambda}^{(p, q)}\left(\left\{P_{i}, Z_{i}\right\}\right)=\frac{\left(\frac{P_{24}}{P_{14}}\right)^{\frac{\Delta_{1}-\Delta_{2}}{2}}\left(\frac{P_{14}}{P_{13}}\right)^{\frac{\Delta_{3}-\Delta_{4}}{2}}}{\left(P_{12}\right)^{\frac{\Delta_{1}+\Delta_{2}}{2}}\left(P_{34}\right)^{\frac{\Delta_{3}+\Delta_{4}}{2}}} \sum_{s=1}^{5} g_{\lambda, s}(r, \eta) Q^{(s)}\left(\left\{P_{i}, Z_{i}\right\}\right),
$$

\footnotetext{
${ }^{5}$ See appendix $\mathrm{F}$ of the companion paper [46] on the relation between projectors and spherical harmonics.
} 
where

$$
\begin{aligned}
& Q^{(1)}=H_{1,3}, \\
& Q^{(2)}=V_{1,23} V_{3,21}, \\
& Q^{(3)}=V_{1,23} V_{3,41}, \\
& Q^{(4)}=V_{1,43} V_{3,21}, \\
& Q^{(5)}=V_{1,43} V_{3,41} .
\end{aligned}
$$

It is then straightforward to write the functions $g_{\lambda, s}$ in terms of the functions $F_{s}$,

$$
\begin{aligned}
& g_{1}(r, \eta)=\mathcal{A} f_{4} F_{5}\left(r^{2}-1\right)^{2} \\
& g_{2}(r, \eta)=\mathcal{A} f_{1}\left(-2 r^{2}\left(f_{4} F_{5}+2 F_{3} \eta r\right)+f_{2}\left(2 F_{4} \eta r^{2}+F_{2}\left(r^{2}+1\right)\right)-2 F_{1}\left(r^{3}+r\right)\right), \\
& g_{3}(r, \eta)=\mathcal{A} f_{1}\left(2 f_{4} F_{5} r+2 \eta\left(2 F_{4} \eta r^{2}+\left(r^{2}+1\right)\left(F_{3} r^{2}+F_{2}\right)\right)+F_{1}\left(r^{2}+1\right)^{2}\right), \\
& g_{4}(r, \eta)=\mathcal{A} f_{1}\left(2 r\left(f_{3} F_{3}+f_{4} F_{5}\right)-f_{2}\left(f_{3} F_{4}+2 F_{2} r\right)+4 F_{1} r^{2}\right), \\
& g_{5}(r, \eta)=\mathcal{A} f_{1}\left(-f_{3}\left(2 F_{4} \eta+F_{3}\left(r^{2}+1\right)\right)-2 f_{4} F_{5}-2 F_{1}\left(r^{3}+r\right)-4 F_{2} \eta r\right),
\end{aligned}
$$

where again we dropped the label $\lambda$ and here the functions $f_{i}$ are given by

$$
\begin{array}{lrl}
f_{1}=r^{2}-2 \eta r+1, & f_{2}=-r^{2}-2 \eta r+1, \\
f_{3}=-r^{2}+2 \eta r+1, & f_{4}=r^{2}+2 \eta r+1,
\end{array}
$$

and

$$
\mathcal{A}(r, \eta)=\frac{2^{\sum_{i} \Delta_{i}}}{\left(1-r^{2}\right)^{2}\left(r^{2}+2 \eta r+1\right)}\left(\frac{r^{2}-2 \eta r+1}{r^{2}+2 \eta r+1}\right)^{\frac{1}{2}\left(\Delta_{12}-\Delta_{34}\right)} .
$$

Now we can already express the partial waves in terms of the function $W_{s}$. The last step is to obtain a recurrence relation for the coefficients $w_{s}$ of the series expansion (3.37).

As we did in the one external vector case we use the Casimir equation to obtain five coupled differential equations involving the functions $W_{s}$. Then we rewrite them in the basis of the expansion in such a way to get a recurrence relation for the coefficients (see appendix A). The result is a set of five coupled recurrence relations

$$
\sum_{s^{\prime}=1}^{5} \sum_{(\widehat{m}, \widehat{j}) \in \mathcal{S}_{s^{\prime}}^{s}} c_{s^{\prime}}^{s}(\widehat{m}, \widehat{j}) w_{s^{\prime}}(m+\widehat{m}, j+\widehat{j})=0, \quad s=1, \ldots, 5 .
$$

As before we represent the sets $\mathcal{S}_{s^{\prime}}^{s}$ in figure 5 . The colored circles are related to the value of $s^{\prime}$ in (3.45), $s^{\prime}=1$ corresponds to the black dot, increasing values of $s^{\prime}$ correspond to larger circles. Each picture represents a different recurrence relation. The recurrence relations (3.45) for $m=0$ reduce to

$$
\begin{gathered}
{\left[c_{(\Delta, j)}-c_{\lambda}\right] w_{1}(0, j)=0,} \\
j\left[c_{(\Delta, j)}-c_{\lambda}\right] w_{2}(0, j)=0, \\
j\left[c_{(\Delta, j)}-c_{\lambda}\right] w_{3}(0, j)=0, \\
j\left[c_{(\Delta, j)}-c_{\lambda}\right] w_{4}(0, j)=0, \\
j\left[c_{(\Delta, j, 1)}-c_{\lambda}\right] w_{5}(0, j)=0 .
\end{gathered}
$$




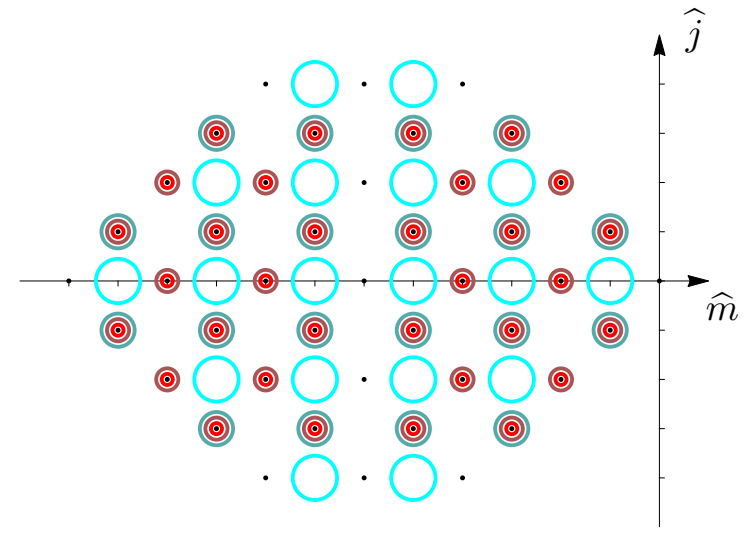

(a) $\mathcal{S}_{s^{\prime}}^{1}$

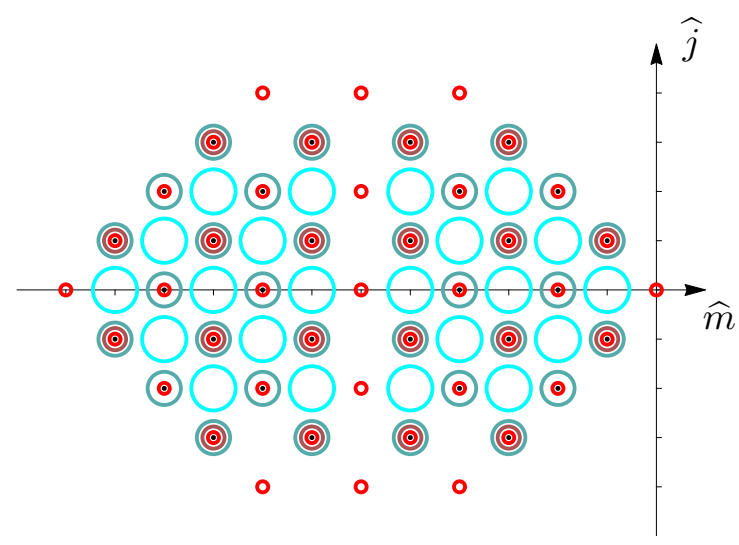

(b) $\mathcal{S}_{s^{\prime}}^{2}$

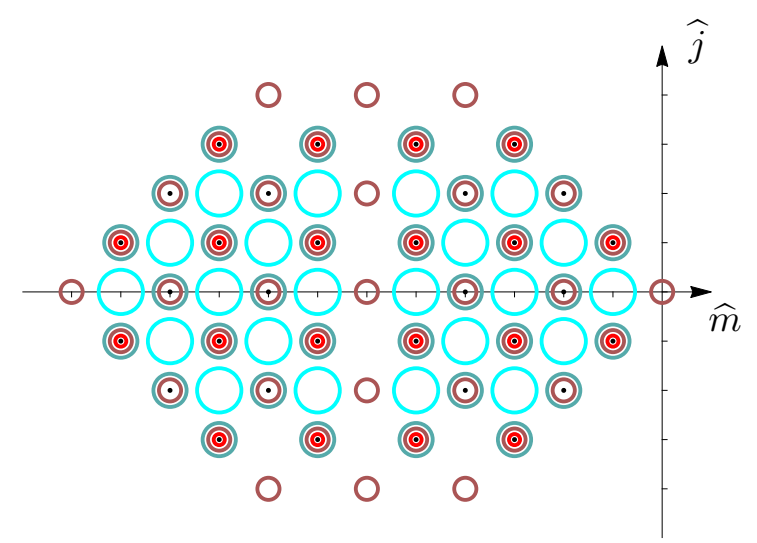

(c) $\mathcal{S}_{s^{\prime}}^{3}$

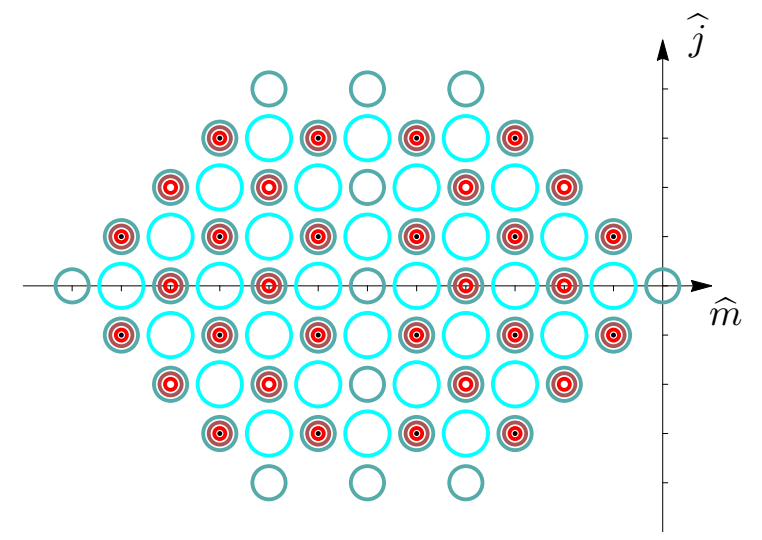

(d) $\mathcal{S}_{s^{\prime}}^{4}$

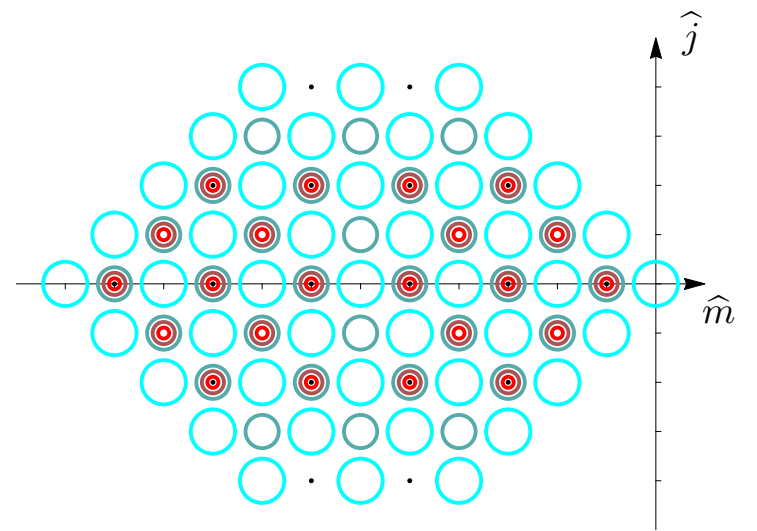

(e) $\mathcal{S}_{s^{\prime}}^{5}$

Figure 5. Pictorial representation of the sets $\mathcal{S}_{s^{\prime}}^{s}$ in formula (3.45). Increasing values of $s^{\prime}$ correspond to increasing radius of the circles. 
The coefficients multiplying $w_{s}(0, j)$ depend on the representation $\lambda$ of the exchanged primary operator. For the exchange of a traceless symmetric representation $\lambda=(\Delta, l)$, it is easy to see that $w_{s}(0, j)=0$ for $j \neq l$. On the other hand, for $j=l$, equations (3.46) only impose $w_{5}(0, l)=0$ leaving $w_{s}(0, l)$ for $s=1,2,3,4$ undetermined. These 4 independent solutions correspond precisely to the 4 independent conformal blocks that we parametrized by $(p, q)$ with $p, q=1,2$. In the case of the representation $\lambda=(\Delta, l, 1)$ we also find $w_{s}(0, j)=0$ for all $j \neq l$. For $j=l$, equation (3.46) implies $w_{s}(0, l)=0$ for $s=1,2,3,4$, and leaves $w_{5}(0, l)$ as the only free parameter. This is consistent with the uniqueness of the conformal block $G_{\Delta, l, 1}$.

It is interesting to notice that equations (3.46) do not constrain $w_{s}(0,0)$ for $s=2,3,4,5$. This should not come as a surprise since the functions $W_{s}$ (with $s=2,3,4,5$ ) defined in (3.39) appear with a derivative in $\eta$ which cancels the term $O\left(\eta^{0}\right)$. Therefore the only coefficients $w_{s}(j, m)$ (with $\left.s=2,3,4,5\right)$ that are physical are the ones with $j>0$.

The coefficients of the expansion of the functions $W_{s}$ have the same structure as shown in figure 2. As an example we want to show the coefficients at level $m=1$ for $j=l+1$. In the case of the conformal blocks $G_{\Delta, l}^{(p, q)}$, these can be found inputting the initial conditions $w_{1}(0, l)=\delta_{p, 1} \delta_{q, 1}, w_{2}(0, l)=\delta_{p, 1} \delta_{q, 2}, w_{3}(0, l)=\delta_{p, 2} \delta_{q, 1}, w_{4}(0, l)=\delta_{p, 2} \delta_{q, 2}, w_{5}(0, l)=0$ and using the recurrence relations (3.45). The result is

$$
\begin{aligned}
& w_{1}(1, l+1)=\frac{(2 h+l-2)\left(l \delta_{p, 2}+\Delta_{12} \delta_{p, 1}\right)\left(l \delta_{q, 2}-\Delta_{34} \delta_{q, 1}\right)}{(h+l-1)(\Delta+l)}, \\
& w_{2}(1, l+1)=\frac{(2 h+l-2)\left(l \delta_{p, 2}+\Delta_{12} \delta_{p, 1}\right)\left(\delta_{q, 1}-\Delta_{34} l \delta_{q, 2}\right)}{(l+1)(h+l-1)(\Delta+l)}, \\
& w_{3}(1, l+1)=\frac{(2 h+l-2)\left(\Delta_{12} l \delta_{p, 2}+\delta_{p, 1}\right)\left(l \delta_{q, 2}-\Delta_{34} \delta_{q, 1}\right)}{(l+1)(h+l-1)(\Delta+l)}, \\
& w_{4}(1, l+1)=\frac{(2 h+l-2)\left(\Delta_{12} l \delta_{p, 2}+\delta_{p, 1}\right)\left(\delta_{q, 1}-\Delta_{34} l \delta_{q, 2}\right)}{(l+1)^{2}(h+l-1)(\Delta+l)},
\end{aligned}
$$

and $w_{5}(1, l+1)=0$. Notice that the $w_{5}(m, j)$ do not vanish in general. For example, $w_{5}(1, l) \neq 0$ because there is a descendant of the primary $\mathcal{O}_{\Delta, l}$ in the representation $(\Delta+1, l, 1)$ constructed by taking one derivative and antisymmetrizing it with an index of the operator. On the other hand, $w_{5}(n, l+n)=0$ for any $n$, because the $n$-th symmetrized derivative constructs a descendant labeled by $(\Delta+n, l+n)$. These features are also evident from figure 5 .

Similarly we can study the block $G_{\Delta, l, 1}$ by setting the initial conditions $w_{5}(0, l)=1$ and $w_{s}(0, l)=0$ for $s=1,2,3,4$. In this case we obtain

$$
w_{5}(1, l+1)=-\frac{\Delta_{12} \Delta_{34} l(2 h+l-3)}{(l+1)(h+l-1)(\Delta+l)},
$$

and $w_{s}(1, l+1)=0$ for $s=1,2,3,4$. Again, by looking at figure 5 one it is clear that $w_{s}(1, l) \neq 0$ (for $s=1,2,3,4$ ). Therefore all the five functions $W_{s}$ contribute to the construction of the conformal block at level $m>0$.

In this case the condition $\Delta_{12}=0=\Delta_{34}$ gives a surprising result. In fact we obtain that many $c_{s^{\prime}}^{s}(\widehat{m}, \widehat{j})$ are zero in such a way that the recurrence relation for $s=1,4,5$ 
are just coupled between them-self (and similarly we find two coupled relations for $s=$ $2,3)$. Another new feature is that the condition $\Delta_{12}=0=\Delta_{34}$ does not imply that the coefficients $c_{s^{\prime}}^{s}(\widehat{m}, \widehat{j})$ with odd $\widehat{m}$ vanish. However they have to respect a specific parity rule: for each recurrence relation if a dot is at a $\widehat{m}$ odd (even), then all the dots of the same color appear at $\widehat{m}$ odd (even). Again these results come just by inspection but it would be interesting to have more analytical insights on why this should be the case (and on weather these are the minimal recurrence relations). Other special cases can be analysed by using the recurrence relations defined in the Mathematica files.

\subsection{Two external spin 2 operators and two scalars}

In this section we study all the conformal blocks appearing the decomposition of a four point function with two spin 2 tensors and two scalar operators,

$$
\begin{aligned}
\left\langle\mathcal{O}_{1}\left(P_{1}, Z_{1}\right) \mathcal{O}_{2}\left(P_{2}\right) \mathcal{O}_{3}\left(P_{3}, Z_{3}\right) \mathcal{O}_{4}\left(P_{4}\right)\right\rangle= & \sum_{\mathcal{O} \in \amalg} \sum_{p, q=3}^{5} c_{12 \mathcal{O}}^{(p)} c_{34 \mathcal{O}}^{(q)} G_{\Delta, l}^{(p, q)}\left(P_{i}, Z_{i}\right) \\
& +\sum_{\mathcal{O} \in \Psi} \sum_{p, q=1}^{2} c_{12 \mathcal{O}}^{(p)} c_{34 \mathcal{O}}^{(q)} G_{\Delta, l, 1}^{(p, q)}\left(P_{i}, Z_{i}\right) \\
& +\sum_{\mathcal{O} \in \boxplus} c_{12 \mathcal{O}} c_{34 \mathcal{O}} G_{\Delta, l, 2}\left(P_{i}, Z_{i}\right) .
\end{aligned}
$$

There are now nine conformal partial waves $G_{\Delta, l}^{(p, q)}($ for $p, q=3,4,5)$ when $\mathcal{O}$ is in the symmetric and traceless representation $(\Delta, l)$, four $G_{\Delta, l, 1}^{(p, q)}$ (for $\left.p, q=1,2\right)$ if $\mathcal{O}$ is in the representation $(\Delta, l, 1)$ and a single one $G_{\Delta, l, 2}$ when $\mathcal{O} \in(\Delta, l, 2)$. As in the two external vector case, we are going to study all the cases at once.

First we study the conformal block $\mathcal{G}_{\lambda}$ on the cylinder and expand it in the allowed polynomial structures $p_{s}$ as described in (3.5). In particular, there can exist 14 possible structures

$$
\begin{array}{ll}
p_{1}=\left(n \cdot z_{1}\right)^{2}\left(n \cdot z_{3}\right)^{2}, & p_{8}=\left(n \cdot z_{1}\right)\left(n^{\prime} \cdot z_{1}\right)\left(n^{\prime} \cdot z_{3}\right)^{2}, \\
p_{2}=\left(n \cdot z_{1}\right)^{2}\left(n \cdot z_{3}\right)\left(n^{\prime} \cdot z_{3}\right), & p_{9}=\left(n^{\prime} \cdot z_{1}\right)^{2}\left(n^{\prime} \cdot z_{3}\right)^{2}, \\
p_{3}=\left(n \cdot z_{1}\right)\left(n \cdot z_{3}\right)^{2}\left(n^{\prime} \cdot z_{1}\right), & p_{10}=\left(z_{1} \cdot z_{3}\right)\left(n \cdot z_{1}\right)\left(n \cdot z_{3}\right) \\
p_{4}=\left(n \cdot z_{1}\right)^{2}\left(n^{\prime} \cdot z_{3}\right)^{2}, & p_{11}=\left(z_{1} \cdot z_{3}\right)\left(n^{\prime} \cdot z_{1}\right)\left(n \cdot z_{3}\right), \\
p_{5}=\left(n \cdot z_{1}\right)\left(n^{\prime} \cdot z_{1}\right)\left(n \cdot z_{3}\right)\left(n^{\prime} \cdot z_{3}\right), & p_{12}=\left(z_{1} \cdot z_{3}\right)\left(n \cdot z_{1}\right)\left(n^{\prime} \cdot z_{3}\right), \\
p_{6}=\left(n^{\prime} \cdot z_{1}\right)^{2}\left(n \cdot z_{3}\right)^{2}, & p_{13}=\left(z_{1} \cdot z_{3}\right)\left(n^{\prime} \cdot z_{1}\right)\left(n^{\prime} \cdot z_{3}\right) \\
p_{7}=\left(n^{\prime} \cdot z_{1}\right)^{2}\left(n^{\prime} \cdot z_{3}\right)\left(n \cdot z_{3}\right), & p_{14}=\left(z_{1} \cdot z_{3}\right)^{2}
\end{array}
$$

therefore

$$
\mathcal{G}_{\lambda}=\sum_{s=1}^{14} F_{s}(r, \eta) p_{s}
$$


To write a nice expansion for the functions $F_{s}$, we study the decomposition of the four point function in eigenstates of the cylinder Hamiltonian. We obtain

$$
\begin{aligned}
\mathcal{G}_{\lambda}=\sum_{m=0}^{\infty} r^{\Delta+} & \sum_{j} \sum_{a}\left[N_{\mathcal{T}}^{2} u_{0} \widetilde{u}_{0}\left(z_{1}\right)_{\mu_{1}}\left(z_{1}\right)_{\mu_{2}}\left(z_{3}\right)_{\nu_{1}}\left(z_{3}\right)_{\nu_{2}} \mathcal{T}_{j}^{\mu_{1} \mu_{2} \nu_{1} \nu_{2}}\left(n, n^{\prime}\right)\right. \\
& +N_{\mathcal{V}}^{2}\left(u_{1} z_{1} \cdot n+u_{2} z_{1} \cdot \nabla_{n}\right)\left(\widetilde{u}_{1} z_{3} \cdot n^{\prime}+\widetilde{u}_{2} z_{3} \cdot \nabla_{n^{\prime}}\right)\left(z_{1}\right)_{\mu}\left(z_{3}\right)_{\nu} \mathcal{V}_{j}^{\mu \nu}\left(n, n^{\prime}\right) \\
& +\left(u_{3}\left(z_{1} \cdot n\right)^{2}+u_{4} z_{1} \cdot n z_{1} \cdot \nabla_{n}+u_{5}\left(z_{1} \cdot \nabla_{n}\right)^{2}\right) \\
& \left.\times\left(\widetilde{u}_{3}\left(z_{3} \cdot n^{\prime}\right)^{2}+\widetilde{u}_{4} z_{3} \cdot n^{\prime} z_{3} \cdot \nabla_{n^{\prime}}+\widetilde{u}_{5}\left(z_{3} \cdot \nabla_{n^{\prime}}\right)^{2}\right) \mathcal{C}_{j}\left(n \cdot n^{\prime}\right)\right]
\end{aligned}
$$

where $u_{s}$ and $\widetilde{u}_{s}$ are coefficients dependent on $j, m$ and $a$. As in the two external vectors case, we chose to factorize out the coefficients $N_{\mathcal{V}}^{2}$ and $N_{\mathcal{T}}^{2}$ in order to obtain simpler formulae. Again one has to fix their value at $m=0$ depending on the possible primary representation $\lambda$ that is exchanged in the conformal block. The tensor harmonic

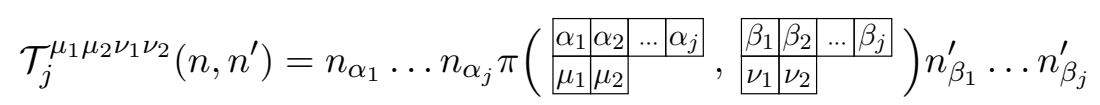

can be written in terms of scalar harmonics as shown in [46]. From this representation it is straightforward to find a set of 14 functions that possess a nice radial expansion, which we can label as follows

$$
W_{p, q}=\sum_{j, m} w_{p, q}(j, m) r^{\Delta+m} \mathcal{C}_{j}(\eta), \quad\left\{\begin{array}{l}
p=q=0 \\
p, q \in\{1,2\} \\
p, q \in\{3,4,5\}
\end{array},\right.
$$

where

$$
w_{p, q}(j, m)=\sum_{a} u_{p}(m, j, a) \widetilde{u}_{q}(m, j, a) .
$$

Next we want to relate the functions $W_{p, q}$ to the conformal partial waves. As usual the first step is to find a relation between $W_{p, q}$ and $F_{s}$ according to (3.51). The result is, for example, ${ }^{6}$

$$
\begin{aligned}
F_{1}= & \partial_{\eta}{ }^{2} W_{3,5}-2 \partial_{\eta}{ }^{2} W_{4,5}+6 \partial_{\eta}{ }^{2} W_{5,5}+\partial_{\eta}{ }^{3} W_{1,2}-3 \partial_{\eta}{ }^{3} W_{2,2}+(3+4(-2+h) h) \partial_{\eta}{ }^{4} W_{0,0} \\
& +\eta\left(-\partial_{\eta}{ }^{3} W_{4,5}+6 \partial_{\eta}{ }^{3} W_{5,5}-\partial_{\eta}{ }^{4} W_{2,2}+\eta \partial_{\eta}{ }^{4} W_{5,5}\right) .
\end{aligned}
$$

We then write the conformal partial wave in terms of the conformal blocks $g_{s}$ using (3.40), where now we have the following allowed structures

$$
\begin{aligned}
& Q_{1}=H_{1,3}^{2}, \quad Q_{8}=H_{1,3} V_{1,4,3} V_{3,2,1}, \\
& Q_{2}=V_{1,2,3}^{2} V_{3,2,1}^{2}, \quad Q_{9}=H_{1,3} V_{1,4,3} V_{3,4,1}, \\
& Q_{3}=V_{1,2,3}^{2} V_{3,4,1}^{2}, \quad Q_{10}=V_{1,2,3} V_{1,4,3} V_{3,2,1}^{2} \text {, } \\
& Q_{4}=V_{1,4,3}^{2} V_{3,2,1}^{2}, \quad Q_{11}=V_{1,2,3} V_{1,4,3} V_{3,4,1}^{2} \text {, } \\
& Q_{5}=V_{1,4,3}^{2} V_{3,4,1}^{2}, \quad Q_{12}=V_{1,2,3}^{2} V_{3,2,1} V_{3,4,1} \text {, } \\
& Q_{6}=H_{1,3} V_{1,2,3} V_{3,2,1}, \quad Q_{13}=V_{1,4,3}^{2} V_{3,2,1} V_{3,4,1} \text {, } \\
& Q_{7}=H_{1,3} V_{1,2,3} V_{3,4,1}, \quad Q_{14}=V_{1,2,3} V_{1,4,3} V_{3,2,1} V_{3,4,1},
\end{aligned}
$$

\footnotetext{
${ }^{6}$ For the sake of brevity the remaining thirteen equations are appended in a Mathematica file.
} 
Therefore we obtain linear relations between the $F_{s}$ and $g_{s}$, for example, ${ }^{7}$

$$
g_{1}=2^{\sum_{i} \Delta_{i}}\left(\frac{r^{2}-2 \eta r+1}{r^{2}+2 \eta r+1}\right)^{\frac{1}{2}\left(\Delta_{12}-\Delta_{34}\right)} F_{14} .
$$

The result of these redefinitions is that the Casimir equation can be written as a set of 14 coupled differential equations on the functions $W_{p, q}$, which we present in a Mathematica file. It is then a tedious exercise to rewrite them as fourteen algebraic relations for the coefficients $w_{p, q}$, as explained in the previous sections. We leave this exercise for the reader.

There are three allowed sets of initial conditions for the coefficients $w_{p, q}$. When the exchanged representation is labeled by $\lambda=(\Delta, l)$ there exist nine conformal blocks $G_{\Delta, l}^{(p, q)}$ with $p, q \in\{3,4,5\}$. We can obtain each block $G_{\Delta, l}^{(p, q)}$ by setting as an initial condition the function $w_{p, q}(0, j)$ (with the same $p$ and $q$ ) to $\delta_{j, l}$ and all the other $w_{p^{\prime}, q^{\prime}}(0, j)$ to zero. In other words, for a given $p, q \in\{3,4,5\}$, the set of fourteen $w_{p^{\prime}, q^{\prime}}(0, j)$ are defined by the initial condition

$$
w_{p^{\prime}, q^{\prime}}(0, j)=\delta_{p^{\prime}, p} \delta_{q^{\prime}, q} \delta_{j, l} .
$$

Similarly, when the exchanged representation is $\lambda=(\Delta, l, 1)$, we have four blocks $G_{\Delta, l, 1}^{(p, q)}$ with $p, q \in\{1,2\}$ which can be obtained by setting

$$
w_{p^{\prime}, q^{\prime}}(0, j)=\delta_{p^{\prime}, p} \delta_{q^{\prime}, q} \delta_{j, l} .
$$

Finally, when we exchange $\lambda=(\Delta, l, 2)$, there exists a single block $G_{\Delta, l, 2}$ which is obtained by setting

$$
w_{p^{\prime}, q^{\prime}}(0, j)=\delta_{p^{\prime}, 0} \delta_{q^{\prime}, 0} \delta_{j, l} .
$$

We checked that these choices of initial condition actually solve the corresponding Casimir equation at lowest order in $r$ and therefore reproduce the correct leading OPE behavior of the four-point function.

\section{Recursion relation from analytic structure in $\Delta$}

In this section we want to use the alternative strategy of [44] in order to obtain a radial expansion for all the conformal blocks that can appear in the conformal block decomposition (3.27) for the four-point function of two vector and two scalar operators.

Following the idea of [13], in [44] a different method to obtain the $r$-series expansion of the conformal blocks is described. The main strategy is to find recurrence relations from the analytic structure of the conformal blocks in the conformal dimension $\Delta$ of the exchanged operator. In [44] the full set of poles $\Delta_{A}^{\star}$ of a bosonic conformal block $G_{\lambda}^{(p, q)}$ for the exchange the most generic representation $\lambda=\left(\Delta, l_{1}, \ldots, l_{[h]}\right)$ was obtained. Moreover, it was explained that the residue at a pole $\Delta_{A}^{\star}$ is just a linear combination of new conformal blocks $G_{\lambda_{A}}^{\left(p^{\prime}, q^{\prime}\right)}$ associated to the exchange of a (null) primary descendant with weight given by $\lambda_{A}=\left(\Delta_{A}, l_{1 A}, \ldots, l_{[h] A}\right)$, namely

$$
G_{\lambda}^{(p, q)} \sim \frac{1}{\Delta-\Delta_{A}^{\star}} \sum_{p^{\prime}, q^{\prime}}\left(R_{A}\right)_{p p^{\prime} q q^{\prime}} G_{\lambda_{A}}^{\left(p^{\prime}, q^{\prime}\right)} .
$$

\footnotetext{
${ }^{7}$ Again the full set of relations is presented in a Mathematica file.
} 
It was also explained how to obtain the coefficients $\left(R_{A}\right)_{p p^{\prime} q q^{\prime}}$ from a direct computation. For concreteness we write the full set of poles $\Delta_{A}^{\star}$ and associated primary descendants $\lambda_{A}$ in the following table

\begin{tabular}{|l|ccc|}
\hline$A$ & $\Delta_{A}^{\star}$ & $n_{A}$ & $l_{k A}$ \\
\hline $\mathrm{I}_{k}, n: n \in\left[1, l_{k-1}-l_{k}\right]$ & $k-l_{k}-n$ & $n$ & $l_{k}+n$ \\
$\mathrm{II}_{k}, n: n \in\left[1, l_{k}-l_{k+1}\right]$ & $2 h+l_{k}-k-n$ & $n$ & $l_{k}-n$ \\
$\mathrm{III}, n: n \in[1, \infty)$ & $h-n$ & $2 n$ & $l_{k}$ \\
$\mathrm{IV}, n: n \in\left[1, l_{[h]}\right]$ & $h+\frac{1}{2}-n$ & $2 n-1$ & $l_{k}$ \\
\hline
\end{tabular}

where $k=1, \ldots,[h]$ and $n$ is an integer. To obtain $\lambda_{A}$ from the data of the table it is enough to know that $\Delta_{A}=\Delta_{A}^{\star}+n_{A}$, and that all the $l_{i A}$ which are not represented in the table are left unchanged $\left(l_{i A}=l_{i}\right.$ for $\left.i \neq k\right)$. We can then reconstruct the full conformal block summing over all the poles in $\Delta$ and over the regular part. In radial coordinates this can be done by writing

$$
g_{\lambda, s}^{(p, q)}(r, \eta)=(4 r)^{\Delta} h_{\lambda, s}^{(p, q)}(r, \eta)
$$

where

$$
h_{\lambda, s}^{(p, q)}(r, \eta)=h_{\infty, \lambda, s}^{(p, q)}(r, \eta)+\sum_{A} \sum_{p^{\prime}, q^{\prime}}(4 r)^{n_{A}} \frac{\left(R_{A}\right)_{p p^{\prime} q q^{\prime}}}{\Delta-\Delta_{A}^{\star}} h_{\lambda_{A}, s}^{\left(p^{\prime}, q^{\prime}\right)}(r, \eta),
$$

and the regular part $h_{\infty, \lambda, s}^{(p, q)}(r, \eta)=\lim _{\Delta \rightarrow \infty} h_{\lambda, s}^{(p, q)}(r, \eta)$ can be computed solving the Casimir equation at leading order in large $\Delta$, as explained in [44]. Given the knowledge of the poles $\Delta_{A}^{\star}$, the coefficients $R_{A}$ and the conformal block at infinity $h_{\infty}$, one can use (4.4) to obtain a series expansion (in $r$ ) for the conformal block. This is usually more efficient than the method of the previous section.

\subsection{Recursion relation for two external vectors and two scalars}

We are now ready to apply the method of [44] to compute all the conformal blocks appearing in the four point function of two external vectors.

Let us start by studying the conformal block $g_{\lambda, s}^{(p, q)}$ for the exchange of a symmetric and traceless representation $\lambda=(\Delta, l)$. The poles $\Delta_{A}^{\star}$ and the residue $g_{\lambda_{A}, s}^{(p, q)}$ which can couple to the external states are characterized by the following table

\begin{tabular}{|c|cccc|}
\hline$A$ & $\Delta_{A}^{\star}$ & $n_{A}$ & $l_{1 A}$ & $l_{2 A}$ \\
\hline $\mathrm{I}_{1}, n: n \in[1, \infty)$ & $1-l-n$ & $n$ & $l+n$ & 0 \\
$\mathrm{II}_{1}, n: n \in[1, l]$ & $l+2 h-1-n$ & $n$ & $l-n$ & 0 \\
$\mathrm{III}, n: n \in[1, \infty)$ & $h-n$ & $2 n$ & $l$ & 0 \\
$\mathrm{I}_{2}, n: n=1$ & 1 & 1 & $l$ & 1 \\
\hline
\end{tabular}


Therefore we can write the following recurrence relation

$$
\begin{aligned}
h_{(\Delta, l), s}^{(p, q)}(r, \eta)= & h_{(\infty, l), s}^{(p, q)}(r, \eta)+\sum_{A} \sum_{p^{\prime}, q^{\prime}=1}^{2}(4 r)^{n_{A}} \frac{\left(R_{A}\right)_{p p^{\prime} q q^{\prime}}}{\Delta-\Delta_{A}^{\star}} h_{\left(\Delta_{A}, l_{A}\right), s}^{\left(p^{\prime}, q^{\prime}\right)}(r, \eta) \\
& +\frac{(4 r)}{\Delta-1}\left(R_{\mathrm{I}_{2}, 1}\right)_{p q} h_{(2, l, 1), s}(r, \eta)
\end{aligned}
$$

where the sum in $A$ runs over $\left(\mathrm{I}_{1}, n\right),\left(\mathrm{II}_{1}, n\right)$ and $(\mathrm{III}, n)$. The coefficients $\left(R_{A}\right)_{p p^{\prime} q q^{\prime}}$ are given in appendix B.1 using the results of [44]. In appendix B.1 we also explain how to find $\left(R_{\mathrm{I}_{2}, 1}\right)_{p q}$, which was not previously computed, ${ }^{8}$

$$
\left(R_{\mathrm{I}_{2}, 1}\right)_{p q}=\frac{1}{2(l+1)(2 h+l-3)}\left(1,-\Delta_{12}\right)_{p}\left(1, \Delta_{34}\right)_{q} .
$$

In appendix B.2 we also compute $h_{(\infty, l), s}^{(p, q)}(r, \eta)$ following the recipe proposed in [44].

When we exchange a representation $\lambda=(\Delta, l, 1)$ we have the following set of poles

\begin{tabular}{|l|cccc|}
\hline \multicolumn{1}{|c|}{$A$} & $\Delta_{A}^{\star}$ & $n_{A}$ & $l_{1 A}$ & $l_{2 A}$ \\
\hline $\mathrm{I}_{1}, n: n \in[1, \infty)$ & $1-l-n$ & $n$ & $l+n$ & 1 \\
$\mathrm{II}_{1}, n: n \in[1, l-1]$ & $l+2 h-1-n$ & $n$ & $l-n$ & 1 \\
$\mathrm{III}, n: n \in[1, \infty)$ & $h-n$ & $2 n$ & $l$ & 1 \\
$\mathrm{II}_{2}, n: n=1$ & $2 h-2$ & 1 & $l$ & 0 \\
\hline
\end{tabular}

We can write the following recurrence relation to determine the block $h_{(\Delta, l, 1)}$

$$
\begin{aligned}
h_{(\Delta, l, 1), s}(r, \eta)= & h_{(\infty, l, 1), s}(r, \eta)+\sum_{A}(4 r)^{n_{A}} \frac{R_{A}}{\Delta-\Delta_{A}^{\star}} h_{\left(\Delta_{A}, l_{A}, 1\right), s}(r, \eta) \\
& +(4 r) \sum_{p, q=1}^{2} \frac{\left(R_{\mathrm{II}_{2}, 1}\right)_{p q}}{\Delta-(d-2)} h_{(d-1, l), s}^{(p, q)}(r, \eta),
\end{aligned}
$$

where again $\sum_{A}$ runs over $\left(\mathrm{I}_{1}, n\right),\left(\mathrm{II}_{1}, n\right)$ and (III, $\left.n\right)$. Since $R_{A}$ are just numbers (not matrices), they can be easily obtained matching the coefficients from the expansion in $r$. We present them in appendix B.1. Moreover in appendix B.1 we also explain how to compute $R_{\mathrm{II}_{2}, 1}$ from first principles,

$$
\left(R_{\mathrm{II}_{2}, 1}\right)_{p q}=\frac{l(h-1)}{2 h+l-2}\left(2 h+l-2, \frac{\Delta_{12}}{l}\right)_{p}\left(2 h+l-2,-\frac{\Delta_{34}}{l}\right)_{q} .
$$

Finally in appendix (B.2) we also find $h_{(\infty, l, 1), s}(r, \eta)$.

A collection of all the definitions needed to obtain the recursion relation for the blocks is presented in a Mathematica file included with the submission.

We would like to comment on an important feature of the recursion relation (4.4). At first sight, the reader might be puzzled by the fact that for most of the poles $\Delta_{A}^{\star}$ of

\footnotetext{
${ }^{8}$ Here the notation $(a, b)_{p}$ means that we pick the component $p$ of the vector $(a, b)$.
} 
formula (4.4) the residue looks divergent because the conformal blocks $G_{\lambda_{A}}^{\left(p^{\prime}, q^{\prime}\right)}$ have a pole precisely at this value of $\Delta$, for every $A$ which is not of the type $\left(\mathrm{II}_{1}, n\right)$ and (III, $\left.n\right)$. In the example above, this phenomena happens in (4.9) for the residue of the block $h_{(\Delta, l, 1), s}$ at the pole $\Delta=(d-2)$. In fact, the term

$$
\sum_{p, q=1}^{2}\left(R_{\mathrm{II}_{2}, 1}\right)_{p q} h_{(d-1, l), s}^{(p, q)}(r, \eta),
$$

in the second line of (4.9), looks divergent because equation (4.6) tells us that $h_{(\Delta, l), s}^{(p, q)}(r, \eta)$ has a pole at $\Delta=d-1$. However, one can easily check that $R_{\mathrm{II}_{2}, 1}$, as defined in (4.10), is such that the combination (4.11) is actually finite. We expect this to happen in general so that, in odd spacetime dimension, the only singularities of the conformal blocks in the $\Delta$-plane are simple poles. Moreover, this analytic structure should extend to non-integer spacetime dimension by analytic continuation. ${ }^{9}$

In this section, we presented the recursion relations (4.6) and (4.9) which allow us to determine all the conformal blocks that can appear in the conformal block decomposition (3.27) of the four-point function with two vector and two scalar operators. In [38], it was shown that the conformal blocks associated to the exchange of symmetric traceless primary operators could be written as differential operators acting on scalar conformal blocks. This gives an alternative way to determine $h_{(\Delta, l), s}^{(p, q)}(r, \eta)$ (see appendix C). On the other hand, $h_{(\Delta, l, 1), s}(r, \eta)$ must be determined using (4.9). Notice that in this context, the last term in (4.9) is a source term that can be written in terms of (derivatives of) the scalar conformal blocks. This is a specific realization of the strategy purposed in [38, 39]. They suggested that one should start by computing seed conformal blocks, which have the simplest external operators that can exchange a primary in a given $\mathrm{SO}(d)$ irreducible representation. For example, the seed blocks for symmetric traceless operators are scalar CBs. Then, one can create external spin by acting with appropriate differential operators $[26,38,39]$. The seed conformal blocks have the advantage that they are unique, in the sense that there is only one block for the chosen external operators. This means that the recursion relations (4.4) are simpler for seed CBs. This is clear in the particular example of equation (4.9). This is just one equation, where the poles are proportional to the same CB with the exception of one pole that can be determined from other seed CBs.

\section{Conclusion}

In the first part of this paper we generalized the series expansion in the radial coordinate introduced by [42] for general CBs in any spacetime dimension. To do so we defined an expansion which inherits the structure of the conformal representations exchanged in the $\mathrm{CB}$. We considered the $\mathrm{CB}$ as a projection of a four-point function obtained by summing over a complete basis of a conformal representation. We organized the sum by grouping together all the descendant operators in the same irreducible representations $\mathbf{Y}$ of $\mathrm{SO}(d)$. Each state gives a contribution proportional to $r^{E}$ where $E$ is the eigenvalue of the cylinder hamiltonian. Therefore, we naturally obtained the $\mathrm{CB}$ in an expansion in radial coordinates. We implemented the method in order to build CBs when the external operators

\footnotetext{
${ }^{9}$ In even spacetime dimension there are higher order poles in the $\Delta$-plane.
} 
are in the traceless and symmetric representation, but one could generalize it for external fermionic operators or for mixed symmetry external operators [40]. We exemplified the method in four cases, for the scalar CB, for one external vector and three scalars, two vectors and two scalars and finally for two spin two operators and two scalars.

Another useful result of this work is to explain how to write closed form recurrence relations for the coefficients of the expansion of the functions $W_{s}(r, \eta)$ that define the CB. These can be obtained by following an algorithm, which we give. On the other hand there can exist infinitely many equivalent recurrence relations and it is less straightforward to find efficient ones. We give recipes to simplify them, however it is still not clear which are the optimal ones and how to find them. We give examples of such simplified recurrence relations in three cases: the scalar $\mathrm{CB}$, one external vector and three scalars, and two vectors and two scalars.

One of the nice features of these relations is that they are analytical results, therefore they can be used to extract many interesting properties of the coefficients of the expansions. For example one can consider explicitly the limit $\Delta \rightarrow \infty$ of the recurrence relations and check that the result is finite. Also, for the scalar block one can trivially check that for $\Delta_{12}=0=\Delta_{34}$, all the coefficients at odd level are identically zero. For the other CBs the condition $\Delta_{12}=0=\Delta_{34}$ is even more interesting: for one external vector and three scalars the two coupled recurrence relations completely decouple, while in the two external vectors case, the five relations form two decoupled groups. Other limit cases, like large $l$ and large $h$, can be also approached analytically using the recurrence relations. The reader is encouraged to play with the Mathematica files in these respects.

The algorithm to build CBs from the recurrence relations is quite efficient for numerical evaluations. However when many parameters (like $h, \Delta_{12}, \Delta_{34}, \Delta, l$ ) are left unfixed, the recurrence relations may become slow. This happens because every coefficient at a certain level is written in terms of a large linear combination of coefficients at lower levels, which are very non trivial rational functions of the parameters. Therefore it may take a long time to simplify the expressions. This problem of course does not subsist when the parameters are numbers, since the simplifications become trivial.

This method has also the feature of generating at the same time the span of all the CBs which can be exchanged in a given four point function. In fact, to compute only one $\mathrm{CB}$, one needs to set to zero all the initial conditions correspondent to the other ones. Because of this feature, it is not straightforward to take advantage of the philosophy of the seed blocks, for which one should study the simplest blocks for a given exchange, and then act with derivatives to obtain more complicated ones. In fact, the relation between the functions $W_{s}$ and the CBs is not trivial since each CB is written as a linear combination of derivatives of $W_{s}$. Therefore the relation between the functions $W_{s}$ of a given $\mathrm{CB}$ and the ones of its seed block takes the form of a complicated differential equation. However, it may be useful to analyze this problem in more detail to find an efficient strategy to include the information about the seed blocks inside the expansion.

The importance of the seed strategy can be easily understood when we consider more complicated four point functions. For example, to study four different spin 2 operators one needs to find the expansion of 633 functions $W_{s}$ which can be obtained from the same 
number of coupled recurrence relations. Even if the technology to do this is ready at the present day (due to the projectors defined in [46]), this strategy is clearly going to be very inefficient. On the other hand the $633 \mathrm{CBs}$ can be obtained from appropriate derivatives of just nine seed blocks which one may derive using a combination of these and other techniques.

It would also be important to find a way of implementing the conservation of external operators in order to decrease the number of functions $W_{s}$ to compute. In fact, the most interesting CBs for numerical bootstrap studies are the ones of four conserved operators, in particular vector currents or stress tensors. In these cases the number of allowed CBs reduces drastically: in general dimensions there are only $7 \mathrm{CBs}$ for four equal vector currents and 29 for four stress tensors [47]. If one could define a single function $W_{s}$ for each CB, the resulting problem would look accessible (surely for the four vector currents case) to the radial expansion even without any further technical development.

In this paper we also applied the alternative strategy of [44], which as well builds the CBs from their radial expansion. The main idea of this method is to study the behavior of CBs in the complex $\Delta$ plane $[13,43]$. From the analysis of [44] we know that the CBs are meromorphic functions of $\Delta$ (in odd dimensions $d$, but it is possible to analytically continue the method to any non even $d$ ), and that the residues at all the poles can always be written as linear combination of other CBs labeled by the position of primary descendants which can appear in the conformal representation. This fact naturally leads to recursion relations. All the poles and the CBs at each residue are known in general while the specific linear combination has to be computed case by case following a systematized algorithm. The regular part of the CBs needs also to be computed case by case and it can be thought as the initial condition for the recursion relation. ${ }^{10}$ The only important subtlety, which was noted in [44], is that the CBs which appear in the residues are actually divergent. This problem was preventing the computation of new CBs using this technology. Here we checked in one explicit case that the divergent CBs at the residue actually appear in a very special linear combination which exactly cancels the divergent piece, giving a finite result. We believe that this should always happen and that this method can be safely used to build any CB.

In particular in this paper we obtained all the five CBs in the scalar-vector-scalarvector four point function: four of them correspond to the exchange of a symmetric and traceless representation, the fifth is a seed block for the exchange of an operator $(\Delta, l, 1)$. The recurrence relation which determines the seed block couples to the other four CBs, but in a linear way. Thus one can simply replace the four CBs by derivatives of the scalar CB (which is the seed block for the exchange of a traceless and symmetric operator). This is an explicit realization of the seed block program. Moreover this structure generalizes for any $\mathrm{CB}$ since the recursion relations of [44] always relate the CBs in a linear way. Therefore in the recurrence relations to determine any seed $\mathrm{CB}$ one will only need to impute derivatives of simpler seed blocks, recursively up to the scalar CB. In addition, as discussed in [44],

\footnotetext{
${ }^{10}$ We believe that also this step can be done in quite general cases; we actually already have this result for four external vectors CBs.
} 
the conservation of external operators can be directly incorporated in this method in order to simplify the equations. And finally, this method allows a more efficient computation of CBs even in the case of many unfixed parameters (like $h, \Delta_{12}, \Delta_{34}, \Delta, l$ ). In fact, at each step of these recurrence relations all the coefficients are known in a closed form, so there is no need to simplify them.

The only downside of this method is that it can be tedious to perform all the computations needed to find the closed form formula for the linear combination of CBs at each pole. Sometimes it may help to have another way to compute the $\mathrm{CB}$ and use it to determine some of these coefficients. In fact this is what we did in the two external vectors case, since we already knew a simpler way to build the CBs from their radial expansion. We therefore believe that the interplay of these two methods can be very profitable in the plan of obtaining spinning conformal blocks.

\section{Acknowledgments}

This research received funding from the [European Union] 7th Framework Programme (Marie Curie Actions) under grant agreements No 269217 and 317089 (GATIS), and from the research grant CERN/FIS-NUC/0045/2015. The work of E.T. has been supported by the Portuguese Fundacão para a Ciência e a Tecnologia (FCT) through the fellowship SFRH/BD/51984/2012. His research was partially supported by Perimeter Institute for Theoretical Physics. Research at Perimeter Institute is supported by the Government of Canada through Industry Canada and by the Province of Ontario though the Ministry of Economic Development \& Innovation.

\section{A Differential equations in Gegenbauer basis}

In the main text we explain how to trade the differential equations for the functions $W_{s}$, which come from the action of the conformal Casimir, with algebraic relations for the coefficients $w_{s}$ of the expansion of the functions $W_{s}$. However, we did not stress that for one differential equation one can in principle write infinite algebraic relations to which would correspond different "dot diagrams" like the ones depicted in figures 4(a) and 4(b). This is not surprising since given a relation as for example (2.27), it is alway possible to sum to it the same relation with shifted $m$ and $j$. The result can still be used as a valid recurrence relation for the coefficients, but it would in general contain more terms and therefore it would be less efficient. The aim of this appendix is to explain the main manipulations which we used to simplify the algebraic relation for the $w_{s}$.

The first caveat is that in general, instead of the last relation of (2.26), it is better to use

$$
\begin{aligned}
\partial_{\eta}^{n} \mathfrak{f}_{m, l}(r, \eta)=\frac{1}{\eta^{2}-1}[ & (5-2 h-2 n) \eta \partial_{\eta}^{n-1} \mathfrak{f}_{m, l}(r, \eta) \\
& \left.+(l-n+2)(2 h+l+n-4) \partial_{\eta}^{n-2} \mathfrak{f}_{m, l}(r, \eta)\right], \quad(n>1)
\end{aligned}
$$

to express any derivative in $\eta$ of order higher than one. Formula (A.1) comes from the usual Gegenbauer differential equation, once we derived it $n-2$ times. This is enough to obtain formula (2.27) for the scalar conformal block. 
For more general cases the procedure is more complicated. In fact, in the scalar case the Casimir equation gives just the single differential equation (2.21), while for any other case we obtain a system of coupled differential equations that we schematically write as $\mathrm{PDE}_{s}=0$ for the set of functions $W_{s}$, where the label $s$ goes from 1 to the total number of tensor structures in the four-point function. Notice that each equation $\mathrm{PDE}_{s}=0$ arises by collecting the terms multiplying the tensor structure $Q^{(s)}$ in the Casimir equation. However, the choice of the basis $Q^{(s)}$ is not unique.

For example, in the case of one external vector the Casimir equation gives rise to a system of two coupled differential equations for the two functions $W_{1}$ and $W_{2}$, that we schematically write as

$$
\operatorname{PDE}_{1}\left(W_{1}, W_{2}\right)=0, \quad \operatorname{PDE}_{2}\left(W_{1}, W_{2}\right)=0 .
$$

We could write the differential equations $\mathrm{PDE}_{s}$ explicitly, but the expressions are very lengthy and they do not provide any deep insight. We want however to stress that both $\mathrm{PDE}_{1}$ and $\mathrm{PDE}_{2}$ can be written as a combination of the terms

$$
\left\{W_{1}, \partial_{\eta} W_{1}, \partial_{\eta} W_{2}, \partial_{\eta}^{2} W_{1}, \partial_{\eta}^{2} W_{2}, \partial_{\eta}^{3} W_{2}, \partial_{r} W_{1}, \partial_{r} \partial_{\eta} W_{2}, \partial_{r}^{2} W_{1}, \partial_{r}^{2} \partial_{\eta} W_{2}\right\}
$$

with appropriate non zero coefficients depending on $r$ and $\eta$, and on the parameters $h$, $\Delta, l, \Delta_{12}$ and $\Delta_{34}$. As a first step, it is natural to try to simplify the system. To do so our criteria was to find new differential equations which involve less terms with high order derivatives. For example, if we ask for which coefficients $a(r, \eta)$ the combination $\mathrm{PDE}_{1}+a(r, \eta) \mathrm{PDE}_{2}$ can be written just in terms of

$$
\left\{W_{1}, \partial_{\eta} W_{1}, \partial_{\eta} W_{2}, \partial_{\eta}^{2} W_{1}, \partial_{r} W_{1}, \partial_{r}^{2} W_{1}\right\}
$$

we obtain that $a(r, \eta)=-\eta$. Similarly, if we want to use the set

$$
\left\{W_{1}, \partial_{\eta} W_{2}, \partial_{\eta}^{2} W_{2}, \partial_{\eta}^{3} W_{2}, \partial_{r} \partial_{\eta} W_{2}, \partial_{r}^{2} \partial_{\eta} W_{2}\right\}
$$

we find the condition $a(r, \eta)=\left(r^{2}+1\right) /(2 r)$. Therefore we obtain a simpler system of equation by choosing

$$
\mathrm{PDE}_{1}-\eta \mathrm{PDE}_{2}=0, \quad 2 r \mathrm{PDE}_{1}-\left(r^{2}+1\right) \mathrm{PDE}_{2}=0 .
$$

Applying this simplification we obtain two coupled algebraic relations $\mathrm{AR}_{s}$ for the coefficients $w_{s}$. The first of which is actually the one defined in (3.24) with $s=1$. The second one can instead be schematically written as

$$
\operatorname{AR}_{2}(m, j) \equiv \sum_{s=1}^{2} \sum_{(\widehat{m}, \widehat{j}) \in \widetilde{\mathcal{S}}_{s}} \widetilde{c}_{s}(\widehat{m}, \widehat{j}) w_{s}(m+\widehat{m}, j+\widehat{j})=0
$$

where the coefficients $\widetilde{c}_{s}$ are in general different from the ones of (3.24) and $\widetilde{\mathcal{S}}_{s}$ is represented in figure 6. Equation (A.7) can still be used to find the coefficients of the expansions of $W_{1}$ and $W_{2}$ even if there are two points with maximal $\widehat{m}$. In fact, one can for example choose 


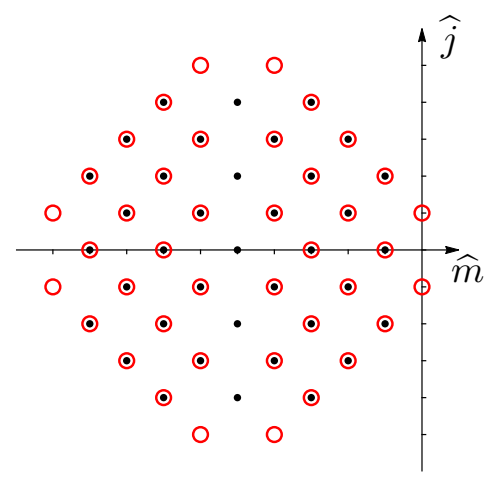

Figure 6. The set $\widetilde{\mathcal{S}}_{s}$ appearing in the linear combination (A.7).

to express $w_{s}(m, j)$ with maximal $m$ and lower $j$ in terms of the other coefficients. But this is not enough, one also needs to assume that the expansion of $W_{s}$ is of the form of figure 2. Therefore, at each level $m$ one should start by expressing $w(m, j)$ starting from $j=l+m$ and decreasing $j$ until $\max (0, l-m)$, and imposing that the coefficients with $j \notin[\max (0, l-m), l+m]$ vanish. However, one can convince oneself that the expansion of figure 2 implies that figure 6 is not minimal, and that there should exist a minimal relation which has just a single dot with maximal $\widehat{m}$. A relation with this feature can actually be obtained as follows. Let us first define a new set of algebraic relations indexed by an integer $n$,

$$
\operatorname{ar}^{(n)}(m, j)=\sum_{k=0}^{n} \operatorname{AR}_{2}(m, j-1-2 k) .
$$

When $n=0$, the "dot diagram" of $\operatorname{ar}^{(0)}(m, j)$, would simply be a shifted version of figure 6 . However, increasing the value of $n$, we found (as a result of a computation) that in the "dot diagram" of $\operatorname{ar}^{(n)}(m, j)$ two distinct shapes of points appear and disentangle one from the other, as pictured in figure 7. In figure 7 we considered values of $n$ up to $n=6$, but increasing $n$ further it is possible to send the lower shape of points at arbitrary large and negative values of $\widehat{j}$. When we use $\operatorname{ar}^{(n)}(m, j)$ with large enough $n$ as a recurrence relation, we can simply forget about the lower shape of points since they are just combinations of coefficients $w_{s}(m, j)$ with $j<0$, which are zero. Since the coefficients of the higher shape do not change by increasing $n$, we can just consider the relation $\operatorname{ar}^{(n)}(m, j)$ for any $n$ with $n \geq 4$ (when the two shapes are really disentangled) and set the lower shape to zero. In this way we obtain the algebraic relation (3.24), which only has one dot with maximal $\widehat{m}$.

When we consider two external vectors more complicated structures can arise but it is enough to use the ideas of this section to simplify the recurrence relations.

\section{B Details on the recursion relation from the analytic structure in $\Delta$}

This appendix is devoted to the computation of all the elements which enter formulae (4.6) and (4.9). In the first section we compute the coefficients $R_{A}$ and in the second section we find the large $\Delta$ behavior of the blocks. Finally, in the last section we perform the leading OPE computation for the blocks in the natural normalization provided by the method of [44]. 


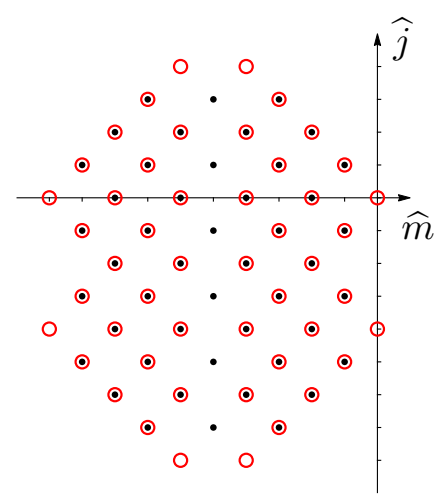

(a) $n=1$

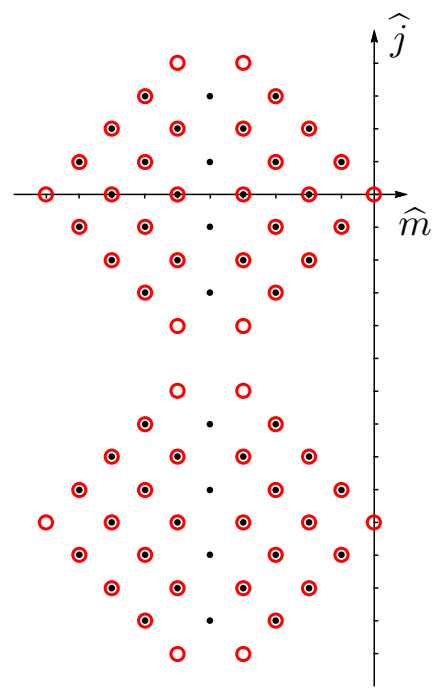

(d) $n=4$

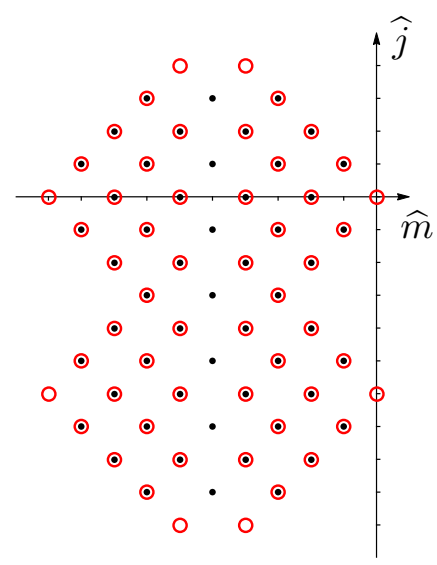

(b) $n=2$

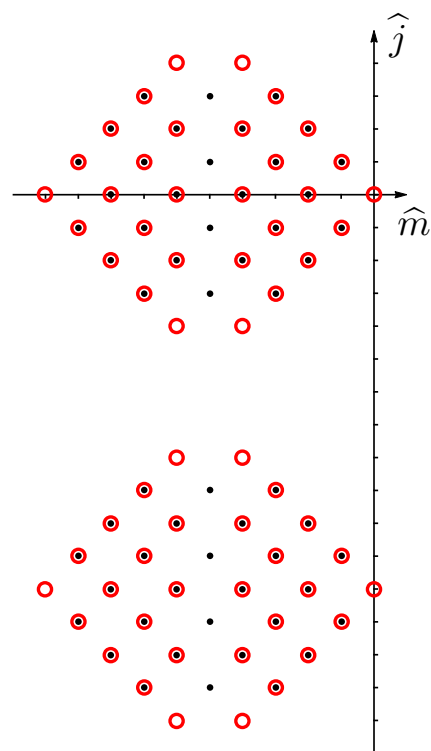

(e) $n=5$

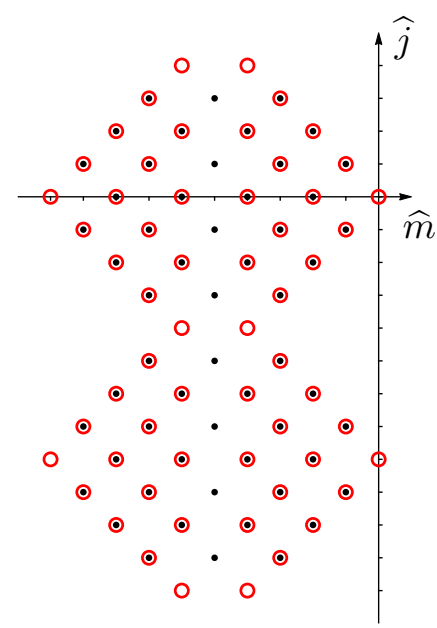

(c) $n=3$

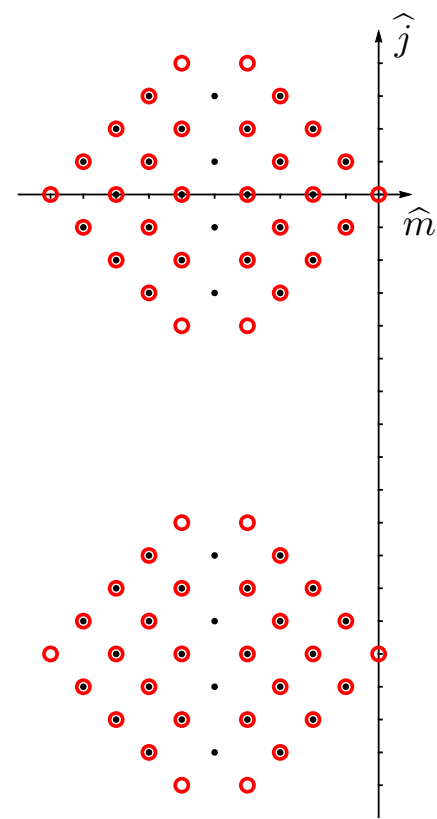

(f) $n=6$

Figure 7. The algebraic relation $\operatorname{ar}^{(n)}(m, j)$. Increasing the value of $n$ it is possible to disentangle two relations.

\section{B.1 The residues $R_{A}$}

In this appendix we want to give more details on the computation of all the coefficients $R_{A}$ in formulas (4.6) and (4.9). We start by a complete derivation of $\left(R_{\mathrm{I}_{2}, 1}\right)_{p q}$ and $\left(R_{\mathrm{II}_{2}, 1}\right)_{p q}$. Then we explain how to obtain $\left(R_{A}\right)_{p p^{\prime} q q^{\prime}}$ of (4.6) from the results of [44]. Finally we write the coefficients $R_{A}$ of (4.9), which we obtained by matching the expansion in $r$.

In the following we obtain from firsts principles the coefficients $\left(R_{\mathrm{I}_{2}, 1}\right)_{p q}$ and $\left(R_{\mathrm{II}_{2}, 1}\right)_{p q}$ of (4.7) and (4.10). Notice that these coefficients arise from new poles which were predicted in [44], but still not observed. These arise from (null) primary descendant states which 
are in a different representation with respect to the primaries. In particular, the primary descendant of type $\mathrm{I}_{2}, 1$ is in the representation $(\Delta+1, l, 1)$, and it is a descendant at the level one of a primary state in the representation $(\Delta, l)$. Conversely, the descendant of type $\mathrm{II}_{2}, 1$ lives in $(\Delta+1, l)$, and is a descendant at the level one of a primary in $(\Delta, l, 1)$. Schematically

$$
\begin{aligned}
& (\Delta, l) \stackrel{\mathrm{I}_{2}, 1}{\longrightarrow}(\Delta+1, l, 1), \\
& (\Delta, l, 1) \stackrel{\mathrm{II}_{2}, 1}{\longrightarrow}(\Delta+1, l) .
\end{aligned}
$$

Using the language of [44], we generate the descendant states acting with an operator $\mathcal{D}_{A}$ on primary states. These descendant states become primary (and therefore null) when the conformal dimension $\Delta$ of the primaries takes specific values,

$$
\begin{array}{rlrl}
\left|\mathrm{I}_{2}, 1\right\rangle & \equiv \mathcal{D}_{\mathrm{I}_{2}, 1}|(\Delta, l) ; z\rangle, & & \text { becomes primary when } \Delta=\Delta_{\mathrm{I}_{2}, 1}^{\star} \equiv 1, \\
\left|\mathrm{II}_{2}, 1\right\rangle \equiv \mathcal{D}_{\mathrm{II}_{2}, 1}|(\Delta, l, 1) ; z, w\rangle, & & \text { becomes primary when } \Delta=\Delta_{\mathrm{II}_{2}, 1}^{\star} \equiv 2 h-2 .
\end{array}
$$

The primary states $|(\Delta, l) ; z\rangle$ and $|(\Delta, l, 1) ; z, w\rangle$ are contracted with polarization vectors as follows

$$
\begin{aligned}
|(\Delta, l) ; z\rangle & \equiv\left|(\Delta, l) ; \underline{\mu_{1}\left|\mu_{2}\right| \ldots \mid \mu_{l}}\right\rangle z^{\mu_{1}} z^{\mu_{2}} \ldots z^{\mu_{l}} \\
|(\Delta, l, 1) ; z, w\rangle & \equiv\left|(\Delta, l, 1) ; \frac{\mu_{1}|\ldots| \mu_{l}}{\nu}\right\rangle z^{\mu_{1}} \ldots z^{\mu_{l}} w^{\nu}
\end{aligned}
$$

and the new operators $\mathcal{D}_{A}$ are

$$
\begin{aligned}
\mathcal{D}_{\mathrm{I}_{2}, 1} & =\frac{-2 i}{(h+l-2)(2 h+l-4)}\left(z_{[\nu} w_{\mu]}\right)\left(P^{[\nu} D_{z, w}^{\mu]}\right), \\
\mathcal{D}_{\mathrm{II}_{2}, 1} & =\frac{-i}{(h-2)(h-1)}\left(P \cdot D_{w, z}\right),
\end{aligned}
$$

where the overall normalizations are chosen for convenience, and $D_{z, w}$ and $D_{w, z}$ are the differential operators which generate projectors to traceless mixed-symmetry tensors [46]. They are defined as

$$
D_{z, w}^{\mu} \equiv d_{00} \partial_{z}^{\mu}+d_{-11} \partial_{w}^{\mu}+z^{\mu} d_{-20}+w^{\mu} d_{-1-1}
$$

where $d_{m n}$ are differential operators with weight $m$ in the variable $z$ and $n$ in the variable $w$,

$$
\begin{aligned}
d_{00} & \equiv(1-h)\left[(2 h-3)+3\left(z \cdot \partial_{z}\right)+\left(w \cdot \partial_{w}\right)\right]-\left(z \cdot \partial_{z}\right)\left(w \cdot \partial_{w}\right)-z^{\alpha}\left(z \cdot \partial_{z}\right) \partial_{z \alpha}, \\
d_{-20} & \equiv \frac{1}{2}\left[2(h-1)+\left(w \cdot \partial_{w}\right)+\left(z \cdot \partial_{z}\right)\right]\left(\partial_{z} \cdot \partial_{z}\right), \\
d_{-11} & \equiv-2(h-1)\left(w \cdot \partial_{z}\right)-\left(w \cdot \partial_{z}\right)\left(w \cdot \partial_{w}\right)-\left(z \cdot \partial_{z}\right)\left(w \cdot \partial_{z}\right), \\
d_{-1-1} & \equiv\left[(h-1)+\left(z \cdot \partial_{z}\right)\right]\left(\partial_{z} \cdot \partial_{w}\right)+\frac{1}{2}\left[\left(w \cdot \partial_{z}\right)\left(\partial_{w} \cdot \partial_{w}\right)-\left(z \cdot \partial_{w}\right)\left(\partial_{z} \cdot \partial_{z}\right)\right],
\end{aligned}
$$

and similarly for $D_{w, z}^{\mu}$ with $z \leftrightarrow w$. 
Using the operators $\mathcal{D}_{A}$ in (B.4) we can proceed to the computation of the coefficients $R_{A}$ as explained in [44]. The following computations are done in a new normalization, with respect to the main text, which is conveniently chosen to apply the techniques introduced in [44]. In the end of the appendix we explain how to return to the conventions used in the main text.

First we compute $Q_{A}$, the residue at the pole $\Delta_{A}^{\star}$ of the inverse of norms $N_{\mathrm{I}_{2}, 1} \equiv$ $\left\langle\mathrm{I}_{2}, 1 \mid \mathrm{I}_{2}, 1\right\rangle$ and $N_{\mathrm{I}_{2}, 1} \equiv\left\langle\mathrm{II}_{2}, 1 \mid \mathrm{II}_{2}, 1\right\rangle$,

$$
\frac{1}{N_{A}} \sim \frac{Q_{A}}{\Delta-\Delta_{A}^{\star}}
$$

This follows from the commutation relations of the conformal algebra, and the result is

$$
Q_{\mathrm{I}_{2}, 1}=\frac{1}{l(l+1)}, \quad Q_{\mathrm{II}_{2}, 1}=\frac{h-1}{2(2 h+l-3)(2 h+l-2)} .
$$

Next we need to obtain the relation between the OPE coefficients of the primary descendant and the OPE coefficients of the original primary. It is convenient to introduce the leading OPE of the three point function of a vector operator $\mathcal{O}_{1}$, a scalar operator $\mathcal{O}_{2}$ and an operator $\mathcal{O}$ in the representations $(\Delta, l)$ and $(\Delta, l, 1)$,

$$
\mathcal{O}(x, z) \mathcal{O}_{1}\left(0, z_{1}\right)=\frac{\mathcal{O}_{2}(0)}{\left(x^{2}\right)^{\alpha_{\Delta} \Delta_{12}}} \times \begin{cases}c_{12 \mathcal{O}}^{(0)} t_{l}^{(0)}\left(x, z, w, z_{1}\right) & \text { if } \mathcal{O} \in(\Delta, l, 1) \\ c_{12 \mathcal{O}}^{(1)} t_{l}^{(1)}\left(x, z, z_{1}\right)+c_{12 \mathcal{O}}^{(2)} t_{l}^{(2)}\left(x, z, z_{1}\right) & \text { if } \mathcal{O} \in(\Delta, l)\end{cases}
$$

with $\alpha_{x} \equiv \frac{\Delta+x+l+1}{2}$ and

$$
\begin{aligned}
t_{l}^{(0)}\left(x, z, w, z_{1}\right) & =\frac{1}{2}|x|(x \cdot z)^{l-1}\left[\left(w \cdot z_{1}\right)(z \cdot x)-\left(z \cdot z_{1}\right)(w \cdot x)\right], \\
t_{l}^{(1)}\left(x, z, z_{1}\right) & =(x \cdot z)^{l}\left(x \cdot z_{1}\right) \\
t_{l}^{(2)}\left(x, z, z_{1}\right) & =(x \cdot z)^{l-1} x^{2}\left(z \cdot z_{1}\right) .
\end{aligned}
$$

Therefore we can define the vectors $M_{\mathrm{I}_{2}, 1}$ and $M_{\mathrm{II}_{2}, 1}$ from the following computation [44]

$$
\begin{aligned}
\mathcal{D}_{\mathrm{I}_{2}, 1} \frac{t_{l}^{(p)}\left(x, z, z_{1}\right)}{\left(x^{2}\right)^{\alpha_{\delta}}} & =\left(M_{\mathrm{I}_{2}, 1}(\delta)\right)_{p} \frac{t_{l}^{(0)}\left(x, z, w, z_{1}\right)}{\left(x^{2}\right)^{\alpha_{\delta}+\frac{1}{2}}}, \quad(p=1,2) \\
\mathcal{D}_{\mathrm{II}_{2}, 1} \frac{t_{l}^{(0)}\left(x, z, w, z_{1}\right)}{\left(x^{2}\right)^{\alpha_{\delta}}} & =\sum_{p=1}^{2}\left(M_{\mathrm{II}_{2}, 1}(\delta)\right)_{p} \frac{t_{l}^{(p)}\left(x, z, z_{1}\right)}{\left(x^{2}\right)^{\alpha_{\delta}+\frac{1}{2}}}
\end{aligned}
$$

The result is

$$
M_{\mathrm{I}_{2}, 1}(\delta)=\left(l, 2 \alpha_{\delta}-2\right), \quad M_{\mathrm{II}_{2}, 1}(\delta)=\left(2 \alpha_{\delta}-1,2 h-2 \alpha_{\delta}+l-1\right) .
$$

The residues are then given by

$$
\begin{aligned}
\left(\widehat{R}_{\mathrm{I}_{2}, 1}\right)_{p q} & =\left.Q_{\mathrm{I}_{2}, 1}\left(M_{\mathrm{I}_{2}, 1}\left(\Delta_{12}\right)\right)_{p}\left(M_{\mathrm{I}_{2}, 1}\left(\Delta_{34}\right)\right)_{q}\right|_{\Delta=1}, \\
\left(\widehat{R}_{\mathrm{II}_{2}, 1}\right)_{p q} & =\left.Q_{\mathrm{II}_{2}, 1}\left(M_{\mathrm{II}_{2}, 1}\left(\Delta_{12}\right)\right)_{p}\left(M_{\mathrm{II}_{2}, 1}\left(\Delta_{34}\right)\right)_{q}\right|_{\Delta=2 h-2} .
\end{aligned}
$$


However, to obtain (4.7) and (4.10) we need to return to the normalization of the conformal blocks used in the main text.

To relate the normalization of the conformal blocks $g_{\lambda}$ of the main text to the normalization of the conformal blocks $\widehat{g}_{\lambda}$ in this appendix, it is convenient to compare their leading OPE behavior. To find the leading OPE for $\widehat{g}_{\lambda}$, we use the techniques proposed in [44] which are extended in appendix B.3. Meanwhile, the leading OPE of $g_{\lambda}$ can be trivially found taking the $O\left(r^{0}\right)$ coefficient of the $r$ expansion according to (3.31) and (3.32). When $\lambda=(\Delta, l)$ we obtain

$$
\widehat{g}_{(\Delta, l), s}^{(p, q)}(r, \eta)=\mathfrak{a}_{l} \sum_{p^{\prime}, q^{\prime}=1}^{2}\left(\mathfrak{m}_{l}^{(L)}\right)_{p, p^{\prime}}\left(\mathfrak{m}_{l}^{(R)}\right)_{q, q^{\prime}} g_{(\Delta, l), s}^{\left(p^{\prime}, q^{\prime}\right)}(r, \eta) .
$$

where

$$
\mathfrak{a}_{l} \equiv \frac{(2 h-1)_{l-1}}{(-2)^{l-1}(h)_{l-1}}, \quad \mathfrak{m}_{l}^{(L)} \equiv\left(\begin{array}{cc}
-1 & 0 \\
-1 & \frac{1}{l}
\end{array}\right), \quad \mathfrak{m}_{l}^{(R)} \equiv\left(\begin{array}{cc}
1 & 0 \\
1 & \frac{1}{l}
\end{array}\right) .
$$

Similarly, when $\lambda=(\Delta, l, 1)$, we have

$$
\widehat{g}_{(\Delta, l, 1), s}(r, \eta)=\mathfrak{b}_{l} g_{(\Delta, l, 1), s}(r, \eta), \quad \mathfrak{b}_{l} \equiv \frac{\mathfrak{a}_{l}}{-2 l(2 h+l-3)} .
$$

Equations (B.14) and (B.16) give rise to the following definition of the coefficients $\left(R_{A}\right)_{p q}$,

$$
\begin{aligned}
\left(R_{\mathrm{I}_{2}, 1}\right)_{p q} & =\left.\mathfrak{a}_{l}^{-1} Q_{\mathrm{I}_{2}, 1} \mathfrak{b}_{l}\left(\left(\mathfrak{m}_{l}^{(L)}\right)^{-1} M_{\mathrm{I}_{2}, 1}\left(\Delta_{12}\right)\right)_{p}\left(\left(\mathfrak{m}_{l}^{(R)}\right)^{-1} M_{\mathrm{I}_{2}, 1}\left(\Delta_{34}\right)\right)_{q}\right|_{\Delta=1}, \\
\left(R_{\mathrm{II}_{2}, 1}\right)_{p q} & =\left.\mathfrak{b}_{l}^{-1} Q_{\mathrm{II}_{2}, 1} \mathfrak{a}_{l}\left(M_{\mathrm{II}_{2}, 1}\left(\Delta_{12}\right) \mathfrak{m}_{l}^{(L)}\right)_{p}\left(M_{\mathrm{II}_{2}, 1}\left(\Delta_{34}\right) \mathfrak{m}_{l}^{(R)}\right)_{q}\right|_{\Delta=2 h-2},
\end{aligned}
$$

which finally gives (4.7) and (4.10), once we replace the values of $Q_{A}$ and $M_{A}$ computed in (B.8) and (B.12).

Similarly, we obtain the coefficients $\left(R_{A}\right)_{p p^{\prime} q q^{\prime}}$ of (4.6),

$$
\left(R_{A}\right)_{p p^{\prime} q q^{\prime}}=\left.\mathfrak{a}_{l}^{-1} Q_{A} \mathfrak{a}_{l_{A}}\left(\left(\mathfrak{m}_{l}^{(L)}\right)^{-1} M_{A}^{(L)} \mathfrak{m}_{l_{A}}^{(L)}\right)_{p p^{\prime}}\left(\left(\mathfrak{m}_{l}^{(R)}\right)^{-1} M_{A}^{(R)} \mathfrak{m}_{l_{A}}^{(R)}\right)_{q q^{\prime}}\right|_{\Delta=\Delta_{A}^{\star}},
$$

where $Q_{A}$ and $M_{A}$ are presented in formulae (34-36) and (189) of [44].

The coefficients $R_{A}$ of (4.9) could in principle be computed using the same technology that we used in this appendix, but we decided to find them matching terms in the expansion in $r$ of the conformal blocks. This can be easily done since these coefficients do not present any matrix structure. Moreover, they are not conceptually new since they arise from null states created by the operators $\mathcal{D}_{\mathrm{I}, n}, \mathcal{D}_{\mathrm{II}, n}$ and $\mathcal{D}_{\mathrm{III}, n}$ already defined in [44]. The result is

$$
\begin{aligned}
R_{\mathrm{I}_{1}, n} & =-\frac{l\left(\frac{-n+\Delta_{12}+1}{2}\right)_{n}\left(\frac{-n+\Delta_{34}+1}{2}\right)_{n}(2 h+l-3)_{n}}{(n-1) ! n !(l+n)(h+l-1)_{n}}, \\
R_{\mathrm{II}_{1}, n} & =-\frac{(-1)^{n}(-l-1)_{n}\left(\frac{-n+\Delta 12+1}{2}\right)_{n}\left(\frac{-n+\Delta 34+1}{2}\right)_{n}(2 h+l-n+1-2)_{n}}{n !(n-1) !(h+l-n+1-1)_{n}(2 h+l-n+1-3)_{n}}, \quad \text { B.19) } \\
R_{\mathrm{III}, n} & =\frac{\left(\frac{h-n+1}{2}\right)_{n}\left(\frac{h-n-2}{2}\right)_{n}\left(\frac{\Delta_{12}+h+l-n}{2}\right)_{n}\left(\frac{\Delta_{12}-h-l-n+2}{2}\right)_{n}\left(\frac{\Delta_{34}+h+l-n}{2}\right)_{n}\left(\frac{\Delta_{34}-h-l-n+2}{2}\right)_{n}}{(-1)^{n+1} 4^{-n} n !(n-1) !(h+l-n)_{2 n}(h+l-n-1)_{2 n}} .
\end{aligned}
$$




\section{B.2 Conformal block at large $\Delta$}

The goal of this section is to find $h_{(\infty, l), s}^{(p, q)}(r, \eta)$ of $(4.6)$ and $h_{(\infty, l, 1), s}(r, \eta)$ of (4.9). They can be obtained solving the leading term in $\Delta$ of the Casimir equation

$$
\mathcal{C} \sum_{p, q} c_{12 \mathcal{O}}^{(p)} c_{34 \mathcal{O}}^{(q)} G_{\lambda}^{(p, q)}\left(\left\{P_{i}, Z_{i}\right\}\right)=c_{\lambda} \sum_{p, q} c_{12 \mathcal{O}}^{(p)} c_{34 \mathcal{O}}^{(q)} G_{\lambda}^{(p, q)}\left(\left\{P_{i}, Z_{i}\right\}\right)
$$

First we replace (3.40) and $g_{\lambda, s}(r, \eta)=(4 r)^{\Delta} h_{\lambda, s}(r, \eta)$ in (B.20), and then we keep the term linear in $\Delta$. We obtain five coupled first order differential equations in the variable $r$ for the functions $h_{\infty, \lambda, s}(r, \eta) \equiv \lim _{\Delta \rightarrow \infty} h_{\lambda, s}(r, \eta)$. We then make the ansatz for the function $h_{\infty, \lambda, s}$,

$$
h_{\infty, \lambda, s}(r, \eta)=\mathcal{A}^{\Delta_{12}, \Delta_{34}}(r, \eta) \sum_{t=1}^{5} F_{s}^{t}(r, \eta) h_{\lambda, t}(0, \eta),
$$

where

$$
\mathcal{A}^{\Delta_{12}, \Delta_{34}}(r, \eta)=\frac{\left(1-r^{2}\right)^{-h-1}}{\left(1+r^{2}-2 r \eta\right)^{\frac{-\Delta_{12}+\Delta_{34}+1}{2}}\left(1+r^{2}+2 r \eta\right)^{\frac{\Delta_{12}-\Delta_{34}+3}{2}}},
$$

and $F_{s}^{t}(0, \eta)=\delta_{s, t}$ so that $h_{\infty, \lambda, s}(0, \eta)=h_{\lambda, s}(0, \eta)$. Using the ansatz (B.21), the five coupled differential equations can be schematically written as

$$
\partial_{r} F_{s}^{t}(r, \eta)=\sum_{s^{\prime}=1}^{5} \mathcal{M}_{s s^{\prime}}(r, \eta) F_{s^{\prime}}^{t}(r, \eta)
$$

where $\mathcal{M}_{s s^{\prime}}$ is a known $5 \times 5$ matrix with entries dependent on $r$ and $\eta$. The label $t=1, \ldots 5$ of $F_{s}^{t}$ parametrize the five independent solutions of (B.23). Even if solving a generic system of five coupled first order differential equations is usually very difficult, in this case we can easily obtain the full solution. This is possible since the choice of the ansatz (B.22) turns the solution $F_{s}^{t}$ into polynomials of $r$ and $\eta$ that can therefore be obtained by expanding (B.23) in series of $r$, and then solving it term by term. The choice of (B.22), which makes this possible, is an educated guess inspired by the large $\Delta$ behavior of the scalar conformal block. The result is

$$
F(r, \eta)=\left(\begin{array}{ccccc}
\left(r^{2}-1\right)^{2}\left(2 r \eta+A_{3}\right) & 0 & 0 & 0 & 0 \\
-2 r^{2} A_{1}^{2} & -A_{1} A_{2} A_{3} & -2 r A_{1} A_{3} & 2 r^{2} \eta A_{1} A_{2} & 4 r^{3} \eta A_{1} \\
-2 r\left(2 \eta r^{3}-r^{2}-1\right) A_{1} & -2 r^{2} \eta A_{1} A_{3} & A_{1} A_{3}^{2} & 4 r^{4} \eta^{2} A_{1} & -2 r^{2} \eta A_{1} A_{3} \\
2 r\left(3 r^{2}-2 \eta r-1\right) A_{1} & 2 r A_{1} A_{2} & 4 r^{2} A_{1} & A_{1} A_{2}^{2} & 2 r A_{1} A_{2} \\
-2 r^{2} A_{1}^{2} & 4 r^{3} \eta A_{1} & -2 r A_{1} A_{3} & 2 r^{2} \eta A_{1} A_{2} & -A_{1} A_{2} A_{3}
\end{array}\right)
$$

where

$$
A_{1} \equiv r^{2}-2 \eta r+1, \quad A_{2} \equiv r^{2}-2 \eta r-1, \quad A_{3} \equiv r^{2}+1 .
$$

Notice that the matrix $F_{s}^{t}$ is independent of the $\mathrm{SO}(d)$ representation of the exchanged operator. This is not surprising since this information only appears in the eigenvalue $c_{\lambda}$ in terms of order $O\left(\Delta^{0}\right)$, which do not contribute at leading order at large $\Delta$. 
We can finally obtain $h_{(\infty, l), s}^{(p, q)}(r, \eta)$ of $(4.6)$, and $h_{(\infty, l, 1), s}(r, \eta)$ of $(4.9)$, simply by taking the appropriate initial conditions for the functions $W_{s}$, which correspond to the blocks $G_{\Delta, l}^{(p, q)}$ and $G_{\Delta, l, 1}$.

\section{B.3 The OPE limit of conformal blocks}

In this appendix we explain how to obtain the leading behavior of the conformal blocks for $r \rightarrow 0$, for the exchange of a generic representation $\lambda$ in the normalization used in [44], which here we denote $\widehat{G}_{\lambda}^{(p, q)}$. To do so, we need to introduce some generic definitions. The leading order (in $x_{12}$ ) OPE between two operators $\mathcal{O}_{1}$ and $\mathcal{O}_{2}$, with conformal dimensions $\Delta_{1}$ and $\Delta_{2}$, and spins $l_{1}$ and $l_{2}$, is

$$
\mathcal{O}_{1}\left(x_{1}, z_{1}\right) \mathcal{O}_{2}\left(x_{2}, z_{2}\right) \approx \frac{\mathcal{O}\left(x_{2}, \mathbf{Y}\right)}{\left(x_{12}^{2}\right)^{\frac{\Delta_{1}+\Delta_{2}-\Delta}{2}}} \sum_{q} c_{12 \mathcal{O}}^{(q)} t^{(q)}\left(-\widehat{x}_{12}, \mathbf{Y}, I\left(x_{12}\right) \cdot z_{1}, z_{2}\right),
$$

where $\mathcal{O}$ is a primary labeled by the conformal dimension $\Delta$ and by a Young tableau $\mathbf{Y}$ of $\mathrm{SO}(d)$, which has at most $[h]$ lines and with $\ell_{i}$ boxes in the $i$-th line filled with the indices $\mu_{1}^{(i)}, \ldots \mu_{\ell_{i}}^{(i)}$. The notation $\widehat{x}^{\mu}$ stands for $x^{\mu} /|x|$ and the index $q$ of $t^{(q)}\left(x, \mathbf{Y}, z_{1}, z_{2}\right)$ labels the possible tensor structures generated by $z_{i}^{\mu}$ and $x^{\mu}$ such that

$$
t^{(q)}\left(\alpha x, \mathbf{Y}, \alpha_{1} z_{1}, \alpha_{2} z_{2}\right)=\alpha^{\ell_{1}+l_{1}+l_{2}} \alpha_{1}^{l_{1}} \alpha_{2}^{l_{2}} t^{(q)}\left(x, \mathbf{Y}, z_{1}, z_{2}\right)
$$

As an example the structures $t^{(q)}$ defined in (B.10) are of this form, where the indices of $\mathbf{Y}$ are contracted with polarization vectors $z$ and $w$. When the set of indices $\mathbf{Y}$ appears twice it means that the respective indices are contracted

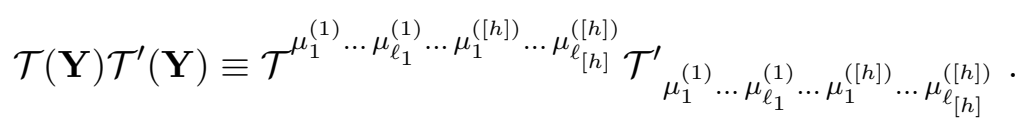

We shall denote by $\pi\left(\mathbf{Y}, \mathbf{Y}^{\prime}\right)$ the projector on the Young tableau labeled by $\mathbf{Y}$. In this notation a two point function of generic operators can be naturally normalized as

$$
\left\langle\mathcal{O}\left(x_{1}, \mathbf{Y}\right) \mathcal{O}\left(x_{2}, \mathbf{Y}^{\prime}\right)\right\rangle=\frac{\pi\left(I\left(x_{12}\right) \cdot \mathbf{Y}, \mathbf{Y}^{\prime}\right)}{\left(x_{12}^{2}\right)^{\Delta}}
$$

where $I\left(x_{12}\right) \cdot \mathbf{Y}$ means that we contract one tensor $I_{\mu \nu}\left(x_{12}\right)$ with each index of the young tableau $\mathbf{Y}$ and where $I_{\mu \nu}(x) \equiv \delta_{\mu \nu}-2 \widehat{x}_{\mu} \widehat{x}_{\nu}$.

We consider the four point function of operators $\mathcal{O}_{i}$ with conformal dimensions $\Delta_{i}$ and spin $l_{i}$, and repeat the reasoning of [44]. Taking the leading OPE (B.26) in the channels $1-2$ and $3-4$, and writing the remaining two point function as (B.29), we obtain

$$
\widehat{G}_{\Delta, l}^{(p, q)} \approx \pi\left(I\left(x_{24}\right) \cdot \mathbf{Y}^{\prime}, \mathbf{Y}\right) \frac{t^{(p)}\left(\widehat{x}_{12}, \mathbf{Y}^{\prime}, I\left(x_{12}\right) \cdot z_{1}, z_{2}\right) t^{(q)}\left(\widehat{x}_{34}, \mathbf{Y}, I\left(x_{34}\right) \cdot z_{3}, z_{4}\right)}{\left(x_{12}^{2}\right)^{\frac{\Delta_{1}+\Delta_{2}-\Delta}{2}}\left(x_{34}^{2}\right)^{\frac{\Delta_{3}+\Delta_{4}-\Delta}{2}}\left(x_{24}^{2}\right)^{\Delta}} .
$$

At the leading order in the limit $x_{12}, x_{34} \rightarrow 0$ we have

$$
x_{13} \approx x_{23} \approx x_{24} \approx x_{14}, \quad(4 r)^{2} \approx \frac{x_{12}^{2} x_{34}^{2}}{\left(x_{24}^{2}\right)^{2}} .
$$


In this limit, we obtain the following formula

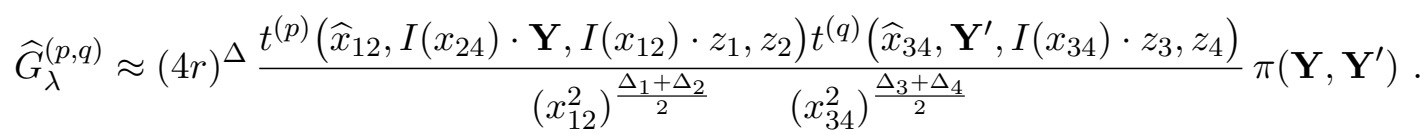

On the other hand the OPE limit of (the hatted version of) (3.16) is

$$
\widehat{G}_{\lambda}^{(p, q)}=\frac{(4 r)^{\Delta}}{\left(x_{12}^{2}\right)^{\frac{\Delta_{1}+\Delta_{2}}{2}}\left(x_{34}^{2}\right)^{\frac{\Delta_{3}+\Delta_{4}}{2}}} \sum_{s} \widehat{h}_{\lambda, s}^{(p, q)}(r, \eta) Q^{(s)},
$$

where we used $\widehat{g}_{\lambda, s}^{(p, q)}=(4 r)^{\Delta} \widehat{h}_{\lambda, s}^{(p, q)}$. We can finally compare (B.33) with (B.32) which gives the main formula to define $\widehat{h}_{\lambda, s}^{(p, q)}(0, \eta)$,

$$
\begin{array}{r}
t^{(p)}\left(\widehat{x}_{12}, I\left(x_{24}\right) \cdot \mathbf{Y}, I\left(x_{12}\right) \cdot z_{1}, z_{2}\right) t^{(q)}\left(\widehat{x}_{34}, \mathbf{Y}^{\prime},\right. \\
\left.\quad I\left(x_{34}\right) \cdot z_{3}, z_{4}\right) \pi\left(\mathbf{Y}, \mathbf{Y}^{\prime}\right) \\
\approx \sum_{s} \widehat{h}_{\lambda, s}^{(p, q)}(0, \eta) Q^{(s)} .
\end{array}
$$

To actually compare the two sides of equation (B.34) we need to expand the left hand side in the structures $Q^{(s)}$ using the fact that $V_{i, j k}, H_{i j}$ and $\eta$, for small $r$, behave as

$$
\begin{aligned}
V_{a, b i} & \approx z_{a} \cdot \widehat{x}_{a b}, & V_{i, j a} & \approx z_{i} \cdot \widehat{x}_{i j}, \\
V_{i, 12} & \approx-z_{i} \cdot I\left(x_{24}\right) \cdot \widehat{x}_{12}, & V_{a, 34} & \approx-z_{a} \cdot I\left(x_{24}\right) \cdot \widehat{x}_{34}, \\
H_{12} & \approx z_{1} \cdot I\left(x_{12}\right) \cdot z_{2}, & H_{34} & \approx z_{3} \cdot I\left(x_{34}\right) \cdot z_{4}, \\
H_{a i} & \approx z_{a} \cdot I\left(x_{24}\right) \cdot z_{i}, & \eta & \approx-\widehat{x}_{12} \cdot I\left(x_{24}\right) \cdot \widehat{x}_{34},
\end{aligned}
$$

where $a, b \in\{1,2\}$ and $i, j \in\{3,4\}$. The only new part of (B.34) with respect to what was discussed in [44] is that we have to implement the contraction of the indices $\mathbf{Y}$. To do so we need to know the projector into the representation labeled by $\mathbf{Y}$.

In the case of the four point function with two vector operators at the points $x_{1}$ and $x_{3}$ and two scalars, we know how to generate the projectors in all the exchanged representations, therefore we can use equation (B.34) to obtain the leading OPE of the conformal blocks. For the case of the symmetric and traceless exchange, formula (B.34) simply reduces to what was already discussed in [44]. Therefore, one can easily obtain $\widehat{g}_{(\Delta, l), s}^{(p, q)}(0, \eta)$ from the definitions of $t_{l}^{(1)}$ and $t_{l}^{(2)}$ in (B.10). Instead, the case of the exchange of $(\Delta, l, 1)$ is new. We can implement the contraction of the indices of (B.34) in two ways, either using the operators $D_{z, w}, D_{w, z}$ of (B.5), or using the closed form for the projector proposed in (3.35). Following the first way we would obtain

$$
\begin{aligned}
& \mathcal{N}_{l, 1} t_{l}^{(0)}\left(\widehat{x}_{12}, I\left(x_{24}\right) \cdot D_{z, w}, I\left(x_{24}\right) \cdot D_{\omega, z}, I\left(x_{12}\right) \cdot z_{1}\right) t_{l}^{(0)}\left(\widehat{x}_{34}, z, \omega, I\left(x_{34}\right) \cdot z_{3}\right) \\
& \approx \sum_{s} \widehat{h}_{(\Delta, l, 1), s}(0, \eta) Q^{(s)}
\end{aligned}
$$

where $t_{l}^{(0)}$ is defined in (B.10). Here we introduced the factor $\mathcal{N}_{l, 1} \equiv \frac{4 l(-1)^{-l-1}}{(l+1) !(h-1)_{l}(2 h-4)_{l+2}}$ in order to properly normalize the projector into the $(l, 1)$ irrep, as shown in [46]. Equivalently, 
we can use the closed form representation (3.35) of the double vector harmonic

$$
\left(I_{\mu}^{\beta}\left(x_{24}\right) I_{\beta \alpha}\left(\widehat{x}_{12}\right) z_{1}^{\alpha}\right)\left(I_{\nu \delta}\left(\widehat{x}_{34}\right) z_{3}^{\delta}\right) \mathcal{V}^{\mu \nu}\left(I\left(x_{24}\right) \cdot \widehat{x}_{12}, \widehat{x}_{34}\right) \approx \sum_{s} \widehat{h}_{(\Delta, l, 1), s}(0, \eta) Q^{(s)} .
$$

From these formulas one can completely fix $\widehat{h}_{(\Delta, l, 1), s}(0, \eta)$ as

$$
\begin{aligned}
& \widehat{h}_{(\Delta, l, 1), 2}(0, \eta)=\widehat{h}_{(\Delta, l, 1), 5}(0, \eta)=c_{l} C_{l-2}^{(h+1)}(\eta), \\
& \widehat{h}_{(\Delta, l, 1), 4}(0, \eta)=\frac{2(1-h)}{l-1} c_{l} C_{l-3}^{(h+1)}(\eta)-\frac{(-2 h-l+3)}{l-1} \widehat{h}_{(\Delta, l, 1), 3}(0, \eta), \\
& \widehat{h}_{(\Delta, l, 1), 3}(0, \eta)=\eta \widehat{h}_{(\Delta, l, 1), 2}(0, \eta), \\
& \widehat{h}_{(\Delta, l, 1), 1}(0, \eta)=\eta \widehat{h}_{(\Delta, l, 1), 4}(0, \eta)-\widehat{h}_{(\Delta, l, 1), 2}(0, \eta),
\end{aligned}
$$

where $c_{l}=-\frac{(-2)^{1-l}(l-1) !}{(2 h+l-3)(h+1)_{l-2}}$.

\section{Differential operators method}

In this appendix we implement the method of [38] to match some of the results obtained from the expansions in radial coordinates presented in the main text. The main idea of [38] is that we can obtain conformal blocks for external operators with spin, by acting with differential operators on the scalar conformal block. Schematically one can write

$$
G_{\Delta, l}^{(p, q)}\left(P_{i}, Z_{i}\right)=D_{\text {Left }}^{(p)} D_{\text {Right }}^{(q)} G_{\Delta, l}\left(P_{i}\right),
$$

where the operators $D_{\text {Left }}^{(p)}$ and $D_{\text {Right }}^{(q)}$ are explicitly defined in [38] in terms of multiplication and derivatives using the embedding vectors $P_{i}$ and $Z_{i}$, and by some shifts of the external dimensions. Using formula (C.1) one can obtain all the blocks for the exchange of a symmetric and traceless representation. Unfortunately, with this method it is not possible to obtain the blocks for more complicated exchanges. However, one can pursue the philosophy of acting with some differential operators on simple seed blocks, which exchange a representation $\lambda$ in order to obtain the most generic blocks labeled by the exchange of the same $\lambda$. This idea was fruitfully followed in four dimensions in [36].

It is instructive to write explicitly how the differential operators look in radial coordinates. To do so we first express the scalar block using equation (2.19) and then collect the coefficients multiplying each tensor structure in the four point function according to (3.16). In the case of the conformal block decomposition (3.2) of the four point function with one external vector, we find

$$
h_{(\Delta, l), s}^{(p)}(r, \eta)=\sum_{q=1}^{2}(\mathcal{M})_{p q} \mathcal{D}_{s}^{(q)} h_{(\Delta, l)}(r, \eta),
$$

where

$$
\mathcal{M}=\frac{-1}{2(\Delta-1)}\left(\begin{array}{cc}
1 & 1 \\
1-\Delta-\Delta_{12} & \Delta-1-\Delta_{12}
\end{array}\right)
$$


and the functions $h_{(\Delta, l)}$ are related to the usual conformal blocks as follows $g_{(\Delta, l)}=$ $(4 r)^{\Delta} h_{(\Delta, l)}$ (and similarly for $h_{(\Delta, l), s}^{(p)}$ ). The matrix $\mathcal{M}$ is introduced in order to match the normalization of the conformal blocks that we used in the main text. The operators $\mathcal{D}_{s}^{(p)}$ are defined as follows

$$
\begin{aligned}
\mathcal{D}_{1}^{(1)} h= & \left(\frac{2\left(1+r^{2}\right)\left(1+\Delta_{12}\right)}{1+r^{2}+2 r \eta}+\frac{1+\Delta+r^{2}\left(-1+\Delta-\Delta_{12}\right)+\Delta_{12}}{-1+r^{2}}\right) h^{[1]} \\
& -\eta \partial_{\eta} h^{[1]}+\frac{\left(r+r^{3}\right) \partial_{r} h^{[1]}}{-1+r^{2}}, \\
\mathcal{D}_{2}^{(1)} h= & \left(-\frac{2 r \Delta}{-1+r^{2}}-\frac{4 r\left(1+\Delta_{12}\right)}{1+r^{2}+2 r \eta}\right) h^{[1]}-\partial_{\eta} h^{[1]}-\frac{2 r^{2} \partial_{r} h^{[1]}}{-1+r^{2}}, \\
\mathcal{D}_{1}^{(2)} h= & \frac{\left(1+r^{2}-2 r \eta\right)\left(-1+\Delta+r^{2}\left(1+\Delta-\Delta_{12}\right)+\Delta_{12}\right) h^{[-1]}}{\left(-1+r^{2}\right)\left(1+r^{2}+2 r \eta\right)} \\
& +\frac{\eta\left(1+r^{2}-2 r \eta\right) \partial_{\eta} h^{[-1]}}{1+r^{2}+2 r \eta}+\frac{\left(r+r^{3}\right)\left(1+r^{2}-2 r \eta\right) \partial_{r} h^{[-1]}}{\left(-1+r^{2}\right)\left(1+r^{2}+2 r \eta\right)}, \\
\mathcal{D}_{2}^{(2)} h= & \frac{2 r\left(2\left(1+\Delta_{34}-\Delta_{12}\right)\left(1-r^{2}\right)-\Delta\left(r^{2}+1-2 r \eta\right)\right) h^{[-1]}}{\left(-1+r^{2}\right)\left(1+r^{2}+2 r \eta\right)} \\
& +\left(-1+\frac{2\left(1+r^{2}\right)}{1+r^{2}+2 r \eta}\right) \partial_{\eta} h^{[-1]}-\frac{2 r^{2}\left(1+r^{2}-2 r \eta\right) \partial_{r} h^{[-1]}}{\left(-1+r^{2}\right)\left(1+r^{2}+2 r \eta\right)},
\end{aligned}
$$

where $h^{[k]}$ means that the variable $\Delta_{12}$ in the function $h$ is shifted by $k$, according to $\Delta_{12} \rightarrow \Delta_{12}+k$. Formula (C.2) is in agreement with the radial expansion of section 3.1 and with the results of [44].

Similarly, for the case of two external vectors we can write $h_{(\Delta, l), s}^{(p, q)}(r, \eta)=$ $\mathcal{D}_{s}^{(p, q)} h_{(\Delta, l)}(r, \eta)$. In this case we do not present the explicit action of the operators since it would be too long. However, we define them in a Mathematica file included with the submission. It is worth mentioning that the result matches the computation of the symmetric and traceless blocks $g_{(\Delta, l), s}^{(p, q)}(r, \eta)$ obtained both from the $r$ expansion and from the analytic structure in $\Delta$.

Open Access. This article is distributed under the terms of the Creative Commons Attribution License (CC-BY 4.0), which permits any use, distribution and reproduction in any medium, provided the original author(s) and source are credited.

\section{References}

[1] S. Ferrara, A.F. Grillo and R. Gatto, Tensor representations of conformal algebra and conformally covariant operator product expansion, Annals Phys. 76 (1973) 161 [INSPIRE].

[2] A.M. Polyakov, Nonhamiltonian approach to conformal quantum field theory, Zh. Eksp. Teor. Fiz. 66 (1974) 23 [INSPIRE].

[3] R. Rattazzi, V.S. Rychkov, E. Tonni and A. Vichi, Bounding scalar operator dimensions in 4D CFT, JHEP 12 (2008) 031 [arXiv:0807.0004] [INSPIRE]. 
[4] V.S. Rychkov and A. Vichi, Universal constraints on conformal operator dimensions, Phys. Rev. D 80 (2009) 045006 [arXiv: 0905.2211] [INSPIRE].

[5] D. Poland and D. Simmons-Duffin, Bounds on $4 D$ conformal and superconformal field theories, JHEP 05 (2011) 017 [arXiv: 1009.2087] [INSPIRE].

[6] R. Rattazzi, S. Rychkov and A. Vichi, Central charge bounds in $4 D$ conformal field theory, Phys. Rev. D 83 (2011) 046011 [arXiv: 1009.2725] [inSPIRE].

[7] R. Rattazzi, S. Rychkov and A. Vichi, Bounds in $4 D$ conformal field theories with global symmetry, J. Phys. A 44 (2011) 035402 [arXiv:1009.5985] [INSPIRE].

[8] D. Poland, D. Simmons-Duffin and A. Vichi, Carving out the space of 4D CFTs, JHEP 05 (2012) 110 [arXiv:1109.5176] [INSPIRE].

[9] S. El-Showk et al., Solving the 3D Ising model with the conformal bootstrap, Phys. Rev. D 86 (2012) 025022 [arXiv:1203.6064] [INSPIRE].

[10] P. Liendo, L. Rastelli and B.C. van Rees, The bootstrap program for boundary $C F T_{d}$, JHEP 07 (2013) 113 [arXiv: 1210.4258] [INSPIRE].

[11] C. Beem, L. Rastelli and B.C. van Rees, The $\mathcal{N}=4$ superconformal bootstrap, Phys. Rev. Lett. 111 (2013) 071601 [arXiv: 1304.1803] [INSPIRE].

[12] F. Gliozzi, More constraining conformal bootstrap, Phys. Rev. Lett. 111 (2013) 161602 [arXiv:1307.3111] [INSPIRE].

[13] F. Kos, D. Poland and D. Simmons-Duffin, Bootstrapping the $O(N)$ vector models, JHEP 06 (2014) 091 [arXiv: 1307.6856] [INSPIRE].

[14] S. El-Showk, M. Paulos, D. Poland, S. Rychkov, D. Simmons-Duffin and A. Vichi, Conformal field theories in fractional dimensions, Phys. Rev. Lett. 112 (2014) 141601 [arXiv: 1309.5089] [INSPIRE].

[15] S. El-Showk et al., Solving the 3D Ising model with the conformal bootstrap II. c-minimization and precise critical exponents, J. Stat. Phys. 157 (2014) 869 [arXiv: 1403.4545] [INSPIRE].

[16] Y. Nakayama and T. Ohtsuki, Approaching the conformal window of $O(n) \times O(m)$ symmetric Landau-Ginzburg models using the conformal bootstrap, Phys. Rev. D 89 (2014) 126009 [arXiv: 1404.0489] [INSPIRE].

[17] S.M. Chester, J. Lee, S.S. Pufu and R. Yacoby, The $\mathcal{N}=8$ superconformal bootstrap in three dimensions, JHEP 09 (2014) 143 [arXiv: 1406.4814] [INSPIRE].

[18] F. Kos, D. Poland and D. Simmons-Duffin, Bootstrapping mixed correlators in the $3 D$ Ising model, JHEP 11 (2014) 109 [arXiv: 1406.4858] [INSPIRE].

[19] M.F. Paulos, JuliBootS: a hands-on guide to the conformal bootstrap, arXiv:1412.4127 [INSPIRE].

[20] C. Beem, M. Lemos, P. Liendo, L. Rastelli and B.C. van Rees, The $\mathcal{N}=2$ superconformal bootstrap, JHEP 03 (2016) 183 [arXiv: 1412.7541] [INSPIRE].

[21] D. Simmons-Duffin, A semidefinite program solver for the conformal bootstrap, JHEP 06 (2015) 174 [arXiv: 1502.02033] [INSPIRE].

[22] F. Gliozzi, P. Liendo, M. Meineri and A. Rago, Boundary and interface CFTs from the conformal bootstrap, JHEP 05 (2015) 036 [arXiv: 1502.07217] [INSPIRE]. 
[23] N. Bobev, S. El-Showk, D. Mazac and M.F. Paulos, Bootstrapping SCFTs with four supercharges, JHEP 08 (2015) 142 [arXiv: 1503.02081] [INSPIRE].

[24] F. Kos, D. Poland, D. Simmons-Duffin and A. Vichi, Bootstrapping the $O(N)$ archipelago, JHEP 11 (2015) 106 [arXiv: 1504.07997] [INSPIRE].

[25] C. Beem, M. Lemos, L. Rastelli and B.C. van Rees, The $(2,0)$ superconformal bootstrap, Phys. Rev. D 93 (2016) 025016 [arXiv: 1507.05637] [INSPIRE].

[26] L. Iliesiu, F. Kos, D. Poland, S.S. Pufu, D. Simmons-Duffin and R. Yacoby, Bootstrapping $3 D$ fermions, JHEP 03 (2016) 120 [arXiv: 1508.00012] [INSPIRE].

[27] M. Lemos and P. Liendo, Bootstrapping $\mathcal{N}=2$ chiral correlators, JHEP 01 (2016) 025 [arXiv: 1510.03866] [INSPIRE].

[28] Y.-H. Lin, S.-H. Shao, D. Simmons-Duffin, Y. Wang and X. Yin, $N=4$ superconformal bootstrap of the K3 CFT, arXiv:1511.04065 [INSPIRE].

[29] S.M. Chester, L.V. Iliesiu, S.S. Pufu and R. Yacoby, Bootstrapping $O(N)$ vector models with four supercharges in $3 \leq d \leq 4$, arXiv: 1511.07552 [INSPIRE].

[30] D. Li, D. Meltzer and D. Poland, Conformal collider physics from the lightcone bootstrap, JHEP 02 (2016) 143 [arXiv: 1511.08025] [INSPIRE].

[31] S.M. Chester and S.S. Pufu, Towards bootstrapping $Q E D_{3}$, arXiv:1601.03476 [INSPIRE].

[32] C. Behan, PyCFTBoot: a flexible interface for the conformal bootstrap, arXiv:1602.02810 [INSPIRE].

[33] D.M. Hofman, D. Li, D. Meltzer, D. Poland and F. Rejon-Barrera, A proof of the conformal collider bounds, JHEP 06 (2016) 111 [arXiv: 1603.03771] [INSPIRE].

[34] F. Kos, D. Poland, D. Simmons-Duffin and A. Vichi, Precision islands in the Ising and $\mathrm{O}(N)$ models, arXiv: 1603.04436 [INSPIRE].

[35] L. Iliesiu, F. Kos, D. Poland, S.S. Pufu, D. Simmons-Duffin and R. Yacoby, Fermion-scalar conformal blocks, JHEP 04 (2016) 074 [arXiv:1511.01497] [INSPIRE].

[36] A. Castedo Echeverri, E. Elkhidir, D. Karateev and M. Serone, Seed conformal blocks in $4 D$ CFT, arXiv: 1601.05325 [INSPIRE].

[37] H. Osborn, Conformal blocks for arbitrary spins in two dimensions, Phys. Lett. B 718 (2012) 169 [arXiv: 1205.1941] [INSPIRE].

[38] M.S. Costa, J. Penedones, D. Poland and S. Rychkov, Spinning conformal blocks, JHEP 11 (2011) 154 [arXiv:1109.6321] [INSPIRE].

[39] A. Castedo Echeverri, E. Elkhidir, D. Karateev and M. Serone, Deconstructing conformal blocks in 4D CFT, JHEP 08 (2015) 101 [arXiv:1505.03750] [INSPIRE].

[40] M.S. Costa and T. Hansen, Conformal correlators of mixed-symmetry tensors, JHEP 02 (2015) 151 [arXiv:1411.7351] [INSPIRE].

[41] F. Rejon-Barrera and D. Robbins, Scalar-vector bootstrap, JHEP 01 (2016) 139 [arXiv: 1508.02676] [INSPIRE].

[42] M. Hogervorst and S. Rychkov, Radial coordinates for conformal blocks, Phys. Rev. D 87 (2013) 106004 [arXiv:1303.1111] [INSPIRE].

[43] A.B. Zamolodchikov, Conformal symmetry in two-dimensions: an explicit recurrence formula for the conformal partial wave amplitude, Commun. Math. Phys. 96 (1984) 419 [INSPIRE]. 
[44] J. Penedones, E. Trevisani and M. Yamazaki, Recursion relations for conformal blocks, arXiv: 1509.00428 [INSPIRE].

[45] M.S. Costa, J. Penedones, D. Poland and S. Rychkov, Spinning conformal correlators, JHEP 11 (2011) 071 [arXiv: 1107.3554] [INSPIRE].

[46] M.S. Costa, T. Hansen, J. Penedones and E. Trevisani, Projectors and seed conformal blocks for traceless mixed-symmetry tensors, arXiv: 1603.05551 [INSPIRE].

[47] A. Dymarsky, On the four-point function of the stress-energy tensors in a CFT, JHEP 10 (2015) 075 [arXiv:1311.4546] [INSPIRE]. 